\title{
A Communications-oriented Perspective on Traffic Management Systems for Smart Cities: Challenges and Innovative Approaches
}

\author{
Soufiene Djahel, Ronan Doolan, Gabriel-Miro Muntean and John Murphy
}

\begin{abstract}
The growing size of cities and increasing population mobility have determined a rapid increase in the number of vehicles on the roads, which has resulted in many challenges for road traffic management authorities in relation to traffic congestion, accidents and air pollution. Over the recent years, researchers from both industry and academia were focusing their efforts on exploiting the advances in sensing, communication and dynamic adaptive technologies to make the existing road Traffic Management Systems (TMS) more efficient to cope with the above issues in future smart cities. However, these efforts are still insufficient to build a reliable and secure TMS that can handle the foreseeable rise of population and vehicles in smart cities. In this survey, we present an up to date review of the different technologies used in the different phases involved in a TMS, and discuss the potential use of smart cars and social media to enable fast and more accurate traffic congestion detection and mitigation. We also provide a thorough study of the security threats that may jeopardize the efficiency of the TMS and endanger drivers' lives. Furthermore, the most significant and recent European and worldwide projects dealing with traffic congestion issues are briefly discussed to highlight their contribution to the advancement of smart transportation. Finally, we discuss some open challenges and present our own vision to develop robust TMSs for future smart cities.
\end{abstract}

Index Terms-Traffic Management System (TMS), Smart Cities, Smart Transportation, Data Sensing and Gathering, VANETs, Route Planning, Traffic prediction.

\section{INTRODUCTION}

$\mathbf{S}$ MART cities is a label that is associated with a significant paradigm shift of interest towards proposing and using various innovative technologies to make cities "smarter" in order to improve the people's quality of life. As a very important and highly visible initiative, the European Commission has launched the European Initiative on Smart Cities in 2010 [1] that addresses four dimensions of the city: buildings, heating and cooling systems, electricity and transport. Strictly related to transportation, the goal is to identify and support sustainable forms of transportation, to build intelligent public transportation systems based on real-time information, Traffic Management Systems (TMS) for congestion avoidance, safety and green applications (e.g. to reduce fuel consumption, gas emissions or energy consumption).

In this context, it is worth noting that the number of cars using the limited road network infrastructure has seen

Soufiene Djahel and John Murphy are with Performance Engineering Laboratory, University College Dublin, Ireland.

Ronan Doolan and Gabriel-Miro Muntean are with Performance Engineering Laboratory, Dublin City University, Ireland.

Manuscript received November 2013 a tremendous growth. One major consequence of this increase is related to management problems that range from traffic congestion control to driving safety and environmental impact. Over recent years, researchers from both industry and academia were focusing their efforts on leveraging the advances in wireless sensing equipment and communication technologies, along with simulation and modeling tools to make the existing road TMS more efficient, enabling them to cope with the above issues in future smart cities. One of the most critical consequence of traffic congestion is the delay of emergency services, such as police, fire and rescue operations, medical services, etc. Indeed, very often individual human lives, general population safety and institutional economic or financial situation in case of incidents, robberies or criminal attacks highly depend on the efficiency and timely response of emergency vehicle services. Additionally, recent road traffic statistics reveal another extremely serious concern which is the increasing number of vehicle crashes. These crashes usually happen in the areas around congested roads as the drivers tend to drive faster, before or after encountering congestions, in order to compensate for the experienced delay. The negative consequences of these accidents are many, at personal, group and societal levels, and could be exacerbated if emergency vehicles are involved in a crash.

However, most large cities in the world are still suffering from traffic congestion, despite employing different solutions to reduce it, including using TMSs deploying advanced congestion control mechanisms. In order to best contribute to the ongoing efforts to solve the traffic congestion problem or at least reduce its impact, there is a need to understand the different types of congestion and their impact. Two major types of congestion can be distinguished: recurrent and nonrecurrent. Recurrent congestion usually occurs when a large number of vehicles use the limited space of the road network simultaneously (e.g. weekday morning and afternoon peak hours). Non-recurrent congestion mainly results from random events such as traffic incidents (e.g. car crash or a stalled vehicle), work zones, bad weather conditions and some special events like sport events, Christmas, etc. According to recent statistics (http://www.transport2012.org), road traffic congestion costs billions to the world economy. For instance losses have reached:

- $200 €$ billions in Europe (2\% of GDP)

- \$101 billion in USA

Aggregate delays of 4.8 billion hours were experienced 
and 1.9 billion gallons of fuel were wasted worldwide. These statistics give a clear indication of the devastating impact that congestion has on individuals, companies (e.g. freight and transport companies, etc.) and society.

Unfortunately, to date the existing TMSs do not provide sufficient and accurate road traffic information to enable granular and timely monitoring and management of the road traffic network. Some of the reasons include: lack of granular data collection, inability to meaningfully aggregate much of the data collected, and a lack of complex management systems capable of providing accurate views of the road transport network. This inability to effectively monitor and manage the traffic maintains traffic congestion high, which in turn affects road safety (i.e. increases the number of death on the roads), augments fuel consumption and causes large gas emissions. The main solutions used by the existing TMSs to manage the traffic after an incident or during peak hours is changing/adapting traffic lights cycles, closing road lanes and intersections, etc. These solutions have limited efficiency when the increasing number of cars are using the limited road infrastructure and constantly new solutions to be used by TMSs are being proposed by the research community.

This survey paper provides a comprehensive study of the solutions employed by existing TMSs, by looking at the different phases of a modern TMS in a smart city environment, from information gathering to service delivery. In particular the paper discusses the Data Sensing and Gathering (DSG) phase in which heterogeneous road monitoring equipment measure traffic parameters (such as traffic volume, speed and road segments occupancy, etc.), and periodically report these readings to a management entity. These monitoring tools can detect random incidents and immediately report them through broadband wireless networks, cellular networks or mobile sensing applications. As these data feeds are fused and aggregated during the Data Fusion, Processing and Aggregation (DFPA) phase to extract useful traffic information, the paper analyses this phase in detail. The Data Exploitation $(\boldsymbol{D E})$ phase uses the acquired knowledge from the data processing phase to compute optimal routes for the vehicles, short-term traffic forecasts, and various other road traffic statistics. Finally in the Service Delivery (SD) phase, the TMS delivers this knowledge to the end users (such as drivers, authorities, private companies, etc.) using a variety of devices such as smart phones, vehicle on-board units, etc. Moreover, the paper investigates the advantages of using alternative approaches, such as mobile sensing and social media, to improve TMS's efficiency and accuracy. This survey also discusses the security attacks that may threaten the integrity of traffic data, leading to non-optimal and incorrect decisions taken by the TMS in relation to the detected/reported incidents. Furthermore, the most significant and recent projects trying to address traffic congestion are briefly discussed, highlighting their contribution to the advancement of TMS. Finally, open challenges are noted and the authors' vision on robust TMS development for future smart cities is presented.

The remainder of this paper is organized as follows. In the next section, we give an overview of future TMSs, highlighting their important conceptual phases and design stages. Then, we address the Data Sensing and Gathering phase with a brief description of the different technologies used for road traffic and events monitoring, and discuss alternative technologies that may improve the quality and accuracy of the collected data. Afterwards, we discuss Data Fusion, Processing and Aggregation techniques, followed by a description of the services that a TMS may provide based on the collected and fused data, including short term traffic prediction information, route planning and parking management information, in sections IV and $\mathrm{V}$, respectively. In section $\mathrm{VI}$, we investigate the different routing approaches used in VANETs to exchange the collected road traffic information among the vehicles, the beacon congestion problem in IEEE 802.11p as well as the simulation tools used for traffic and VANET-based application simulation. Subsequently, we show how smart vehicles may significantly improve the efficiency of current TMSs, in section VII. In section VIII, we discuss the different threats that may jeopardize the security and privacy of TMSs. In section IX, we present the major international projects aiming at improving the different aspects of future TMSs. In the final sections, our vision on open challenges is discussed and this survey paper is concluded.

\section{Overview of Future Traffic Management SYSTEMS}

A Traffic Management System (TMS) offers capabilities that can potentially be used to reduce road traffic congestion, improve response time to incidents, and ensure a better travel experience for commuters. A typical TMS consists of a set of complementary phases, as shown in Figure 1, each of which plays a specific role in ensuring efficient monitoring and control of the traffic flow in the city. The cornerstone phase of a TMS is Data Sensing and Gathering (DSG) in which heterogeneous road monitoring equipment measure traffic parameters (such as traffic volumes, speed and road segments occupancy, etc.), and periodically report these readings to a central entity. For example, these monitoring tools can detect random incidents and immediately report them through wireless networks, cellular networks or mobile sensing applications. Subsequently, these data feeds are fused and aggregated during the Data Fusion, Processing and Aggregation (DFPA) phase to extract useful traffic information. The next phase, Data Exploitation (DE), uses this acquired knowledge from the processed data to compute: optimal routes for the vehicles, short term traffic forecasts, and various other road traffic statistics. Finally in the Service Delivery (SD) phase, the TMS delivers this knowledge to the end users (such as drivers, authorities, private companies, etc.) using a variety of devices such as smart phones, vehicles' on-board units, etc.

The capabilities offered by a TMS are not confined to serve drivers and road authorities only, but can also contribute significantly to the economic growth of a country, to the preservation of citizens' safety and to the support of national security. The currently deployed technologies for road traffic surveillance still suffer from a lack of traffic parameters measurement accuracy and real-time report of events that occur on the roads, 


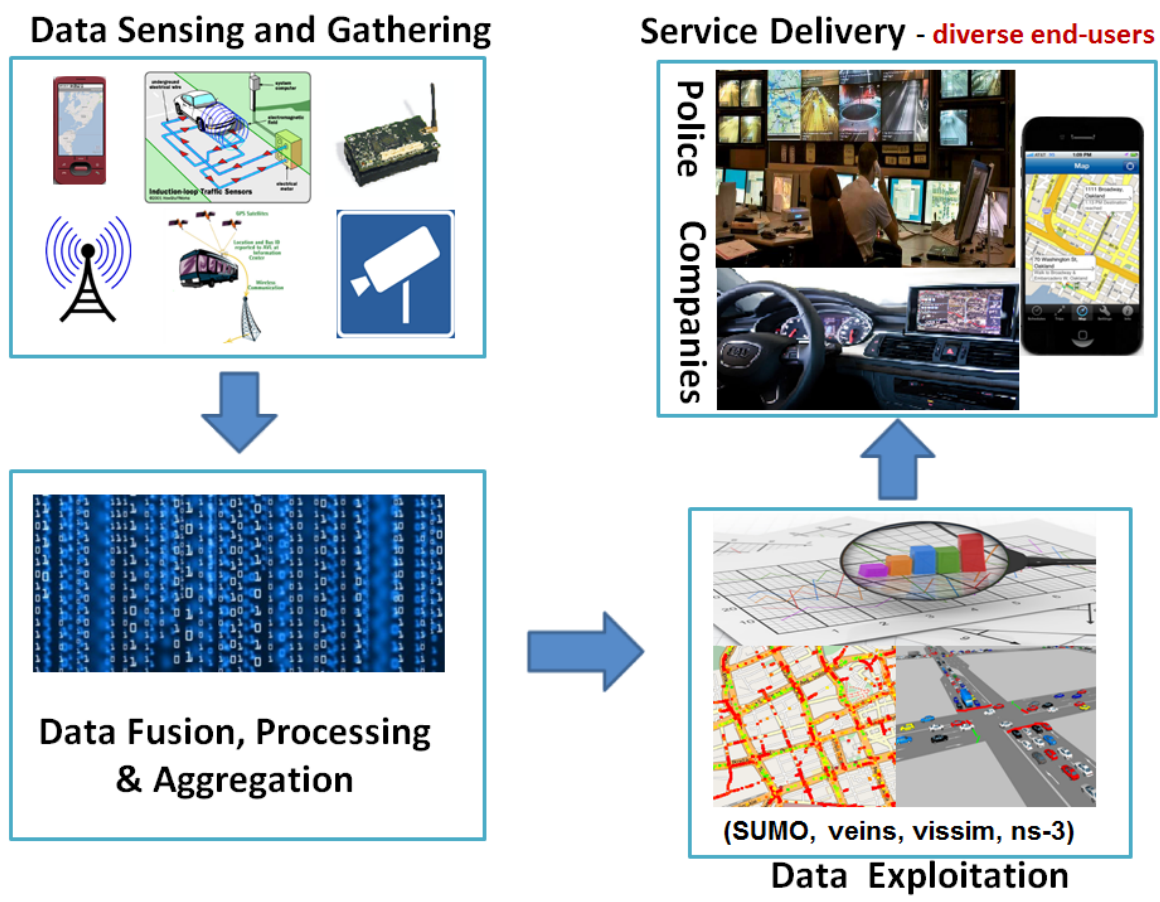

Figure 1: Data life cycle in smart transportation

especially in developing countries. Moreover, the gathered traffic data usually needs to undergo a filtering process to improve its quality and eliminate the noise. Deploying highly sophisticated equipment to ensure accurate estimation of traffic flows and timely detection of emergency events may not be the ideal solution, due to the limitation in financial resources to support dense deployment and the maintenance of such equipment, in addition to their lack of flexibility. Therefore, alternative cost-effective and flexible solutions are needed to guarantee better management of road traffic in both developed and developing countries.

A modern TMS aims to overcome some of the above limitations by designing innovative approaches able to exploit advanced technologies to efficiently monitor the evolving critical road infrastructure. These approaches should be scalable enough in order to enable better control of the traffic flow and enhanced management of large cities' road networks. This will certainly improve the accuracy of the acquired real-time traffic information and the short-term traffic prediction. This will enable making and using short-term predictions based on current traffic volumes to identify bottlenecks and make more informed decisions about how to best reroute traffic, change lane priorities, modify traffic light sequences, etc. A modern TMS should also provide a visual tool that can display in real-time traffic information related to location of bottlenecks, incidents, and congestion level in each road segment, as well as estimated travel time from one location to another in the road network. In this way, the transport authorities will have an overall view of the road network in real-time, and will enable the best support for improvements in the traffic flow management and more efficient reactions to emergency incidents on the roads.

An adequate TMS for future smart cities should fulfill the following requirements:

- Ensure higher accuracy in estimating traffic conditions and better efficiency in dealing with emergency situations on the roads, compared to the existing TMSs.

- Be able to efficiently manage the traffic in road networks of varying size and characteristics.

- Provide real-time road traffic simulation and visualisation to help authorities more efficiently manage the road infrastructure and improve route planning for commuters.

- Ensure simplified and smooth integration of existing systems and new technologies, and manage the evolution of these systems.

A high level architectural overview of a modern TMS is depicted in Figure 2. This figure shows the main components of the TMS needed to deliver the collected road traffic information to the intended end consumers (e.g. road authorities, Police, drivers etc). As we can see from this figure, the core system of the TMS collects road traffic information from heterogeneous data sources according to the consumer needs and specific requests. These data feeds are then aggregated and stored in an unified format in one or multiple databases. Later, upon reception of a consumer request, the core system processes the request and extracts the pertinent data from the appropriate database. Then the requested information is sent back to the intended consumer, tailored for their specific purposes: e.g. analysis and statistics, decision-making, etc.

\section{TRAFFIC DATA SENSING AND GATHERING}

Data sensing and gathering phase focuses on the scalable collection of traffic flow information from a large number of 


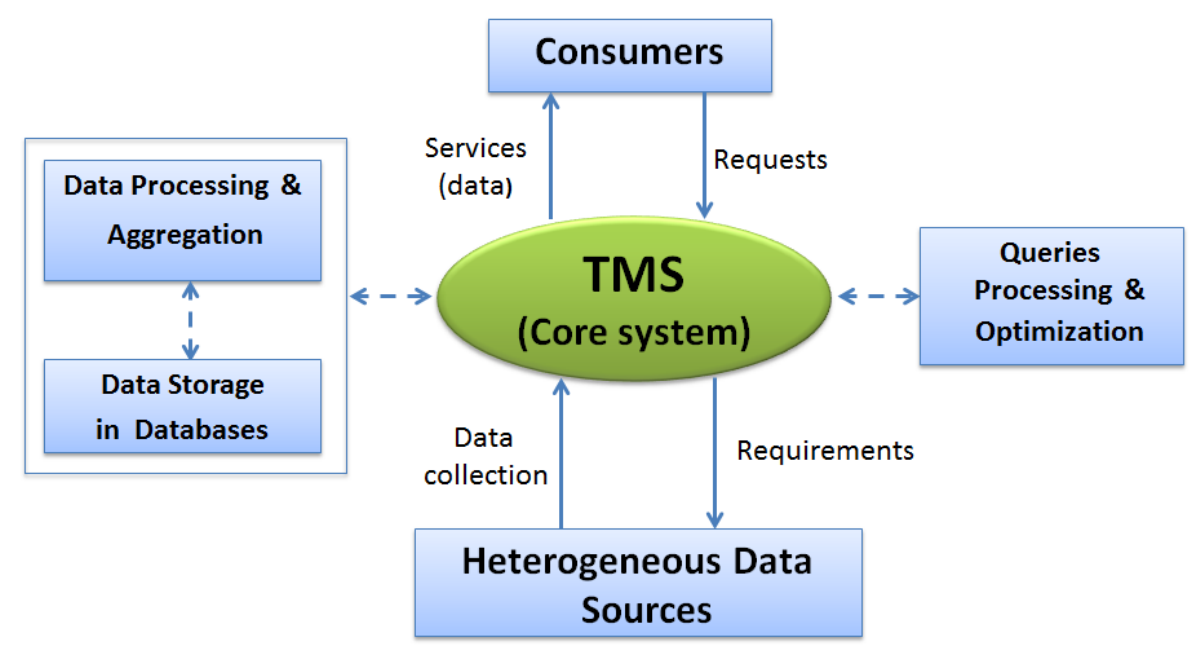

Figure 2: A simple architecture of a modern TMS

heterogeneous sources. Many of the current deployed systems used by traffic management agencies collect data in a variety of formats, time scales, and granularity. This is due to the fact that those systems have been deployed at different time periods with little or no integration between them. This creates a management problem for operators whom must manage, analyse and interpret all of this dissimilar data. A modern TMS will analyse a number of the existing traffic information collection mechanisms employed by city authorities and identify where new technologies and systems can be used to improve the accuracy, timeliness and cost efficiency of data collection. In addition, these new data collection technologies must provide a more informed explanation of the root causes behind the increasing congestion levels on the roads. More specifically, the current trends in TMS development consist in leveraging advanced communication and sensing technologies like Wireless Sensor Networks (WSNs), cellular networks, mobile sensing and social media feeds as potential solutions to circumvent the limitation of the existing systems.

The main wireless technology used for events sensing and gathering on the roads is the tiny sensor devices. These sensors could be mounted on vehicles, at the roadside or under the road pavement to sense and report different events. In the former case, the in-vehicles embedded sensors monitor and measure several parameters related to the vehicle operations and communicate them to the nearby vehicles or roadside units. In the latter cases, the sensors are mainly used to measure the passing vehicles' speed, the traffic volumes as well other environmental parameters. WSNs can be used to interconnect these sensors and greatly reduce the cost of monitoring systems deployment. In an urban scenario, we can imagine a plethora of sensors being deployed to collect data about traffic conditions, air pollution, environmental noise and many other applications. Information can also be obtained from vehicles that have proper sensors and communication antennas on board; these would primarily be public transportation vehicles, taxis, police cars, and freight vehicles. A modern TMS will, therefore, focus on designing innovative solutions able to collect data from a specific region of interest under specific time constraints, while minimising cost and spectrum usage and maximising system utilisation.

\section{A. Wireless Sensor Networks (WSNs)}

Due to their high efficiency and accuracy in sensing the different events, wireless sensors have been widely deployed in various environments for data collection and monitoring purposes [76], [77]. Indeed, it is foreseen that WSNs can enable several applications that may significantly improve the control of road traffic flow and ease its management, examples of these applications are the real-time control of traffic lights [73] and their adaptation according to the congestion level [74], as well as parking spaces management [72]. However, the deployment of wireless sensors in the road environment to realize these applications face several challenges, in addition to the well-known issues in WSNs [75], that require careful consideration and design of appropriate protocols. Among these challenges, we highlight the need of a fast and reliable MAC access protocol [31] and data forwarding mechanisms to guarantee timely transmission of critical messages carrying information about the occurred emergency events on the road. An example of WSNs deployment for road traffic monitoring is shown in Figure 3.

It is also worth mentioning that the expected wide and dense deployment of wireless sensors on the roads necessitates the design of robust data aggregation techniques to deal with the high redundancy and correlation of the transmitted information, especially from neighboring sensors, as the redundant transmission of this information may lead to quick depletion of sensors battery, increase the delay of emergency messages, as well as the collision rate. To reduce traffic data redundancy, the optimal placement of wireless sensors on road networks should be investigated and a trade-off solution between the number of sensors deployed in a specific area, and road events detection and accuracy should be designed. The spatial and temporal correlation of traffic data are intrinsic characteristics of road networks, which can be leveraged to solve both sensor 
data aggregation and optimal sensors placement problems in future smart cities.

\section{B. Machine to Machine (M2M) communication}

A key technology that is a promising solution for reliable and fast traffic data monitoring and collection is Machine to Machine (M2M) communication. The M2M technology has recently attracted increasing attention from both academic and industrial researchers aiming to foster its application for data collection in various environments. Recent forecasts [116], [117] indicate an outstanding market growth over the next few years for M2M devices usage and connectivity. According to these forecasts, billions of devices will be potentially able to benefit from the M2M technology. The report published by the Organisation for Economic Co-operation and Development (OECD) in [118] reveals that around 5 billion mobile wireless devices are currently connected to mobile wireless sensor networks, and foresees that this number will grow to reach 50 billion connected devices by the end of the decade. In M2M communication, a sensor gathers traffic data and sends it via wireless communication/cellular/3G/LTE networks towards one or multiple central servers for processing purposes. The ability of M2M devices to avoid the multi-hop transmission, as opposed to WSNs, makes the data transmission faster and more reliable, which represents a significant benefit for the sensors reporting delay critical events. Moreover, it is foreseeable that this technology will significantly enhance the accuracy of data collection and lead to more flexible deployment of sensors on the roads.

M2M over LTE networks is expected to be a key aspect of future TMS. These M2M devices are equipped with access technology capable of communicating in a reliable, fast and extremely efficient way with the central entity that processes and aggregates the collected data. Moreover, M2M solutions support different classes of QoS, thus they can efficiently collect prioritized data from multiple sources and ensure that appropriate QoS is applied to each stream. The M2M technology provides an extremely attractive solution for data collection in urban areas due to its management benefits in terms of reduced data reporting delay, high efficiency, and low complexity. However, deploying M2M devices as an alternative of WSNs technology will incur an additional cost related to the use of cellular/3G/LTE networks. Therefore, this may hinder the wide deployment of M2M technology by city traffic managers, especially for cities with limited financial resources, which is the case of the majority of cities in developing countries.

\section{Mobile sensing}

In addition to the above data sources, mobile sensing using mobile devices is expected to enable fast detection of events on the roads and enhance the accuracy of traffic conditions monitoring. According to recent studies in [32] and [33], mobile crowd-sensing systems have been recently used to provide more accurate real-time traffic information on a large scale, using smart phones that enable services such as, accurate localization of vehicles, faster and more precise reporting of incidents and accurate travel time estimation for improving commuters travel experience. The key enabler of the widespread of mobile sensing applications, mainly for traffic monitoring purposes, is the voluntary participation of the users. These users demand high level of privacy, anonymity and security guarantees in order to participate to such a system. Indeed, these requirements constitute major concerns that need to be carefully addressed to instigate larger participation of mobile devices users to mobile sensing applications. These issues can be dealt with as discussed in the following to mitigate their impact on the TMS efficiency and accuracy of its decisions.

- Trust management of mobile sensing data sources: how to build a trust relationship with the mobile sensing data source? In this case, reputation systems, such as [144], need to be used to continually assess the level of trustworthiness of each mobile sensing data source. A mobile data source is deemed trustworthy if the information it has reported has been validated by either other mobile sources or a trusted data source such as road-side sensors, induction loops or CCTV cameras.

- Privacy preservation of mobile devices users: several levels of privacy could be defined in the context of smart cities, and users can adjust the setting of their devices to increase/decrease the privacy level according, for example, to traffic conditions (e.g. normal driving conditions, traffic jam, incident ) and the service they need to request from the TMS (e.g. optimal/fastest route to their destination). Therefore, adaptive privacy protection techniques that manage the users privacy preferences and adapt the privacy level to the contextual factors in smart cities are required.

- Design robust authentication techniques to prevent any misuse of the system such as identity spoofing and fake alerts, etc.

\section{Social media}

In the context of smart cities, social media feeds, such as Twitter and Facebook for instance, can play an important role in improving the accuracy and richness of the traffic information provided by the traditional monitoring equipment such as road sensors and induction loops. Despite the fact that these pieces of equipment can measure the vehicles' speed and road segments' occupancy to enable the estimation of traffic congestion level, they are unable to identify the root event that has led to this situation. [70] has shown that relying on social media feeds, in addition to the traditional data sources, can significantly enrich the real-time perception of traffic conditions in the cities, and help to explain the reasons behind the variation of the congestion level. Indeed, revealing the real causes of the sudden increase of the congestion level (e.g. accident, road works, political or social protest etc) will enable more appropriate reaction from the road authorities to alleviate the impact of this situation. Therefore, there is a need to deeply investigate [71] this traffic data source to enhance citizens' quality of life and aid the traffic authorities for efficient management of the increasing number of cars. 


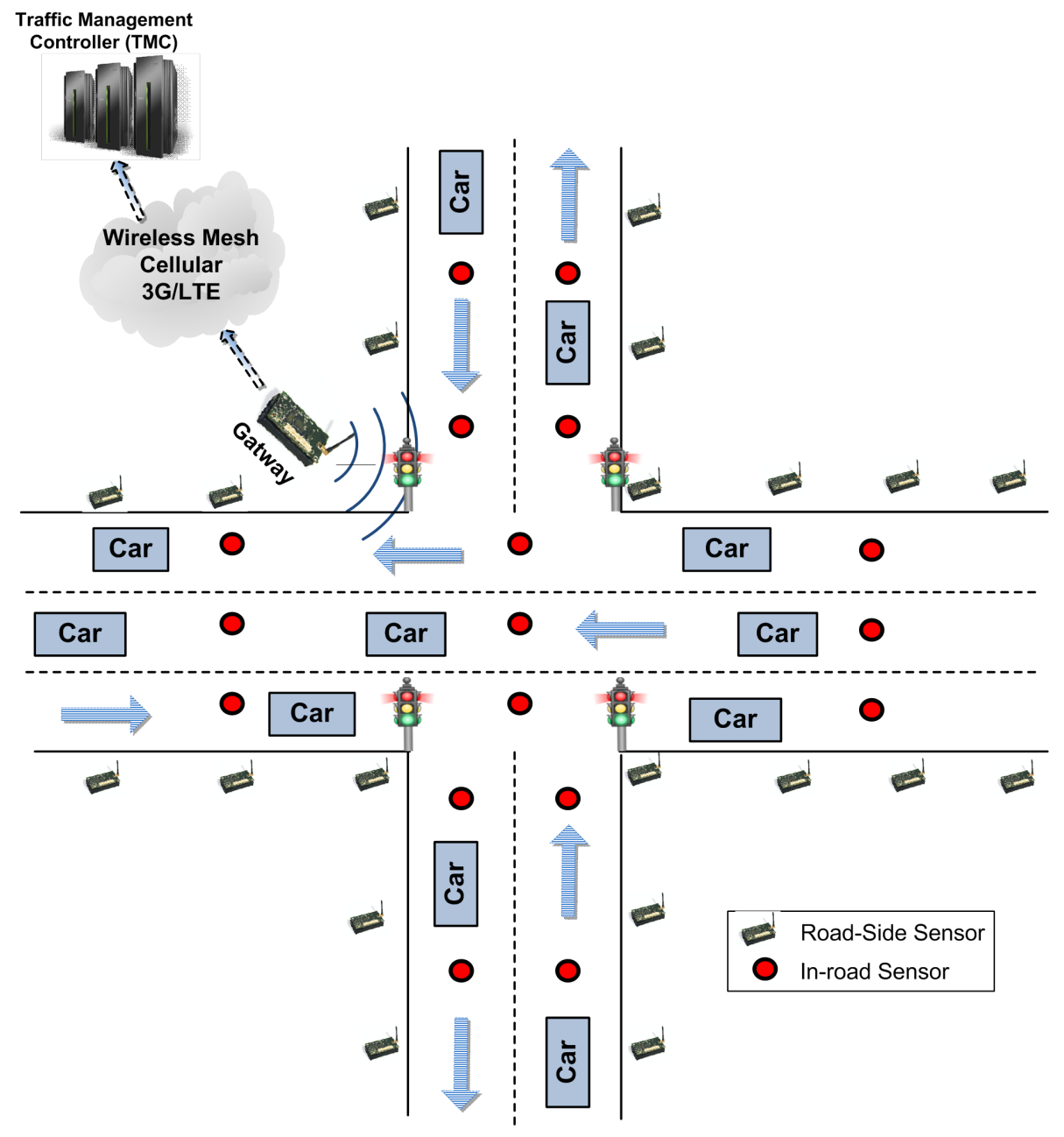

Figure 3: Scenario illustrating wireless technology deployment in road environment for data sensing and gathering

In order to maximize the benefits of using this novel traffic data source, we need to raise the citizens awareness to its utility. Applying a reward system, for example, to encourage the citizens to use social networks to report accidents and unusual events that occur in the roads is highly recommended. In this case, any citizen who reports an authentic emergency/non emergency event will get a reward which will increase their rank among road users. Higher ranked users could get higher quality of service from the TMS. For example, when they sign up to the TMS to plan a trip they will get the route that satisfies all their requirements, while other drivers may just get a route that satisfies a subset of their preferences. Using social media feeds may also assist the road authorities for better planning of road networks expansion, as well as optimal road signs placement and speed limits setting. This is feasible by analyzing the citizens' feedback, including drivers and pedestrians, which may significantly improve traffic flow control and improve road safety.

However, at the same time, there is a need to verify the accuracy of the data acquired from such poorly reliable sources of information. Mechanisms are needed to be proposed and deployed which best balance the need for fast information propagation with validation and verification of both the source of data and the content accuracy. At least an indication of the level of trust in the data is required to be present in order for any further processing to make use of it in an informed manner. To this end, some recent efforts have been devoted to design robust security and privacy preservation solutions. In [138], the authors have investigated the various hacking techniques that may threaten the reliability of such data sources, and presented potential mitigation methods. This paper highlights the dangers incurred by poor security, such as identity theft and corporate espionage etc, and proposes novel architecture to mainly improve the security of personal data. On the other hand, [139] has shown the potential security and privacy challenges that may arise as a consequence of the emergence of MMSN (Multimedia-oriented Mobile Social Network) concept. MMSN is a new social media application in which users in the vicinity share useful multimedia content of interest, such as road traffic information etc. However, this application may create new security threats such as privacy disclosure. The authors presented three MMSN applications emphasising their corresponding security and privacy problems, and discussed a set of solutions to face those threats. The studied applications were mainly content query, service evaluation, and content filtering. In addition to the above works, other researchers 
have also thoroughly investigated privacy concerns and trust management issues in social networks such as [148], [149] and [147].

\section{Data Fusion, Processing And Aggregation TECHNIQUES}

Although there are a large number of systems currently employed for road traffic monitoring, there is very little integration between these systems and in most cases the data from each system has different types, formats and metadata. Much of the data is also of different time scales and levels of granularity. During the DFPA phase of TMS numerous techniques are applied to combine these heterogeneous data sources to produce unified metrics that can be processed and delivered to various consumers based on their requirements. A modern TMS should enable the real-time aggregation of these high volume data sets from a plethora of heterogeneous sources. It will also store this data over long periods of time to perform statistical analysis, which can be further used to better plan and deploy changes/upgrades to the transportation network. This will enable the system to combine the various traffic measurements produced by existing traffic systems such as induction loop counters, CCTV cameras and cellular handover information - to monitor and manage traffic flow within the city.

The main steps involved in DFPA phase are summarized in Figure 4 which describes the processing flow of the data and what are the different issues that this phase deals with. After receiving the gathered data, the DFPA engine applies cleansing and verification techniques to identify incorrect, inaccurate and incomplete data and either correct or remove them. Afterwards, these data will be prepared to the fusion phase by resolving time synchronization issues and exploiting the geographical correlation of these data to further reduce their amount or extract new knowledge. Subsequently, the chosen fusion algorithm is thus applied to integrate the different set of data into a consistent, accurate and valuable representation of the road network traffic. The output of this phase will be then transmitted to the core TMS system and samples of the forwarded data will be stored for future aggregation and redundancy removal purposes.

In order to enable the TMS to scale to larger cities, the deployed techniques must be capable of aggregating traffic data feeds from various levels and at various levels of granularity. For example, a modern TMS will investigate how traffic feeds can be aggregated and filtered for specific geographic regions before being passed to the core system. This reduces the amount of information processing and filtering that is required at the core, and will allow the system to scale and evolve over time and to be deployed to cover increasingly large geographic regions.

Due to the heterogeneity of the collected traffic data formats, a common data format is required to enable high level management and processing of the aggregated data. IBM intelligent transportation product, for example, uses the Traffic Management Data Dictionary (TMDD) standard developed by the Institute of Transportation Engineers (ITE). This standard describes the data concepts for traffic data, metadata, network devices and events. Moreover, IBM Intelligent transportation product uses TMDD standard to ease interfacing with Traffic Management Centers and Advanced Traffic Management Systems (ATMS). The aim of the TMDD standard is to provide a standards-based, high-level definition in a protocol independent manner, with which a system specification interface can be prepared. Besides its main purpose, which is supporting traffic management applications, all ITS practical areas can benefit from TMDD format such as for emergency situation management, products shipment and travel information for communication needs.

The ultimate objective of the introduction of TMDD standard and the development of data fusion and integration techniques is to simplify and automate data collection from existing and future systems, and reduce data aggregation and conversion delay and complexity in order to improve the overall system efficiency. To this end, recent research studies have designed innovative techniques to ensure efficient fusion and integration of the traffic data gathered from heterogeneous road monitoring equipment. A snapshot of these recent works is given below.

The authors of [99] have developed a technique to improve the quality of detector data which is combined with Floating car data (FCD). It is acknowledged that discovering the dynamic properties of the traffic is a difficult task due to the sparseness of induction loops and low penetration rates of vehicles transmitting FCD. To overcome this issue, the authors have used conventional spatio-temporal interpolation to determine fine structures, such as stop and go waves from the collected data. Moreover, interpolation can also be used to compensate for detector failure. The efficiency of this approach has been evaluated using real traffic dataset collected from the roads of Birmingham which are known by the high penetration rate of induction loops.

A more recent technique has been proposed in [100], where ASDA (Automatische Staudynamikanalyse: Automatic Tracking of Moving Jams)/FOTO (Forecasting of traffic objects) model has been applied to process induction loops data, and then fuse the resulting data with FCD. Both of these datasets are processed to determine traffic state changes (e.g. from free flow to congested flow, stopped to congested flow etc). Subsequently, they are fused to construct a spatiotemporal map of the traffic state changes. This work has shown that a probe vehicles penetration rate of $1.5 \%$ has yielded a very similar model to detectors deployed at every $1-2 \mathrm{~km}$, hence the assertion is that now they can be easily combined, resulting in a more efficient traffic model than the original ASDA /FOTO model.

Fuzzy rough set theory has been also used in [101] to fuse heterogeneous traffic data feeds, each of which can often yield contradictory evidence. By using this theory, a significant reduction of the redundant data can be achieved. In addition, a novel fusion technique based on Yagers formula [105] has been developed to rank the different data sources. Furthermore, the maximum fuzzy probability function is applied for the different datasets to avoid the subjective factor effect. It is worthwhile to notice that this work is different from the two 


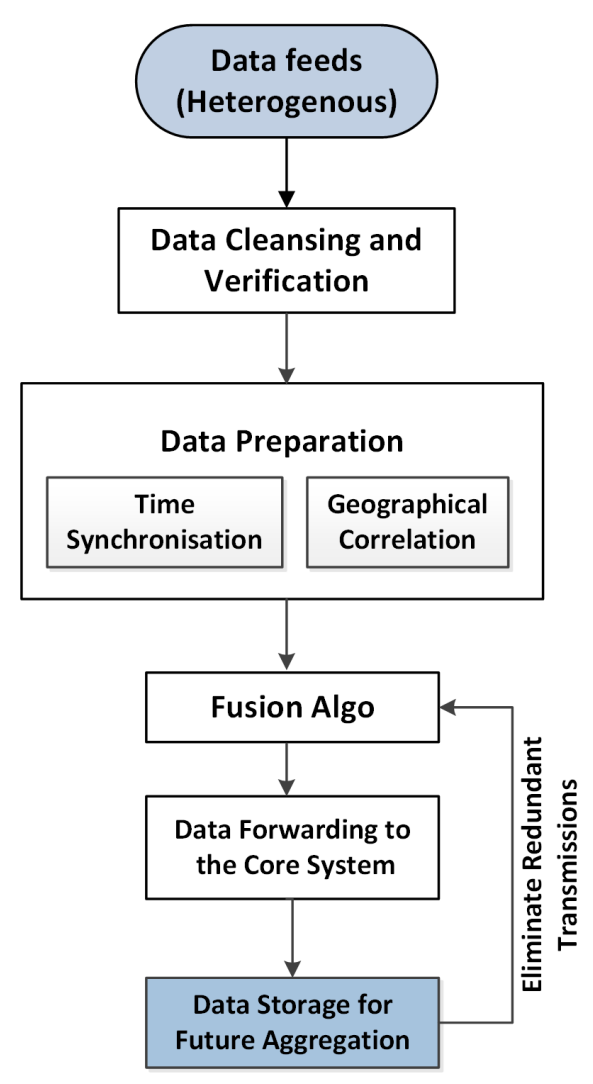

Figure 4: The different steps in DFPA phase

previous techniques as it considers induction loops, video detectors and OD analysers instead of induction loops and floating car data. The efficiency of the developed fusion technique is tested against traffic data from Hangzhou city in China, and the obtained results have proven its effectiveness.

\section{TMS SERVICES}

As shown in Figure 1, after fusing and aggregating the collected data, the TMS can exploit it to provide various services. These services are mainly vehicles routing to shorten the commuter journey, traffic prediction that enables early detection of bottlenecks and more informed decisions to face these issues, parking management systems that ensure optimal usage of the available spots and interact with routing and prediction services for improved control of traffic flow, and finally infotainment services that provide useful information (e.g. tourism information, multimedia contents delivery over VANTEs, shopping centers offers, cinema, ...) for both drivers and passengers. In the following, we will discuss the importance of these services and how their efficiency can be improved to optimize the traffic in the transportation system.

\section{A. Short-term traffic prediction}

Traffic forecasting research topic has been widely investigated in several academic studies aiming at outlining the key factors that influence its design and modelling decisions. In general, the following three factors characterize a short-term traffic forecasting system [102]. First, its scope which refers to whether the forecasting model will be implemented as part of a TMS or a Traveller Information System (TIS), and the area where it will be used (e.g. highway, urban arterials etc). The second factor concerns data resolution which is highly dependent on the chosen forecasting horizon and step. The horizon refers to the extent of time ahead for which the traffic conditions will be predicted, while the step defines the time interval upon which the prediction is made, as stated in [102]. The forecasting accuracy is strongly related to the choice of the horizon and step values, hence defining appropriate time interval for both of them is compulsory to achieve accurate prediction. According to the experiments conducted in [103] the prediction accuracy is inversely proportional to the forecast horizon duration. The Highway Capacity manual (2000), as well as some studies in the literature, have suggested $15 \mathrm{~min}$ as the most appropriate horizon value. For the step value, the most used value is $5 \mathrm{~min}$ interval due to the high variability of the traffic flow. However, we argue that the horizon and step values should be also adapted to the requirements of the application for which the forecasting algorithm will be used. Finally, the third factor that affects the forecasting accuracy is the technique used to model the traffic data, such as statistical and time series analysis models [108] [109], the well-known Auto-Regressive Integrated Moving Average (ARIMA) model [104] [107], Neural Networks [106], pattern recognition techniques, etc. In addition, some recent works have used spatio-temporal correlation of traffic flow across the road network [58], as well as the collected GPS data [61] to provide more precise prediction.

Accurate real-time road traffic forecasting is a required capability to avail of advanced smart transportation technologies. From the point of view of transport authorities, the ability to predict traffic pattern evolution is a key requirement to enable efficient management of the traffic flow in urban, sub-urban and highway scenarios. Traffic prediction can enable the early identification of traffic jams, which allows the traffic authorities to take preventive measures to alleviate the congestion on the roads. On the other hand, traffic prediction is a substantial step towards providing accurate journey duration estimation for the commuters, as it is one of the major inputs of route planning algorithms, as highlighted in section V-B.

In the last decade, numerous traffic prediction techniques have been proposed in the literature; however, most of them have been devoted to highways rather than highly congested urban arterials, which are far more likely to be monitored by the traffic authorities. Therefore, the design of accurate and scalable traffic prediction tools for urban road networks is required. To achieve this goal, the efforts should be focused on defining real-time forward looking analysis techniques that use the large stores of historical traffic data, the social media feeds as well as real-time traffic feeds (i.e. traffic flow, road segments occupancy and speed) to predict how traffic conditions will evolve in time-frames ranging from the next few minutes to a couple of hours, as illustrated in Figure 5. It is worth noting that social media feeds are substantial input as they provide a comprehensive explanation of the traffic conditions, which significantly helps the traffic managers to take the adequate actions. The key principle of a prediction algorithm is to 


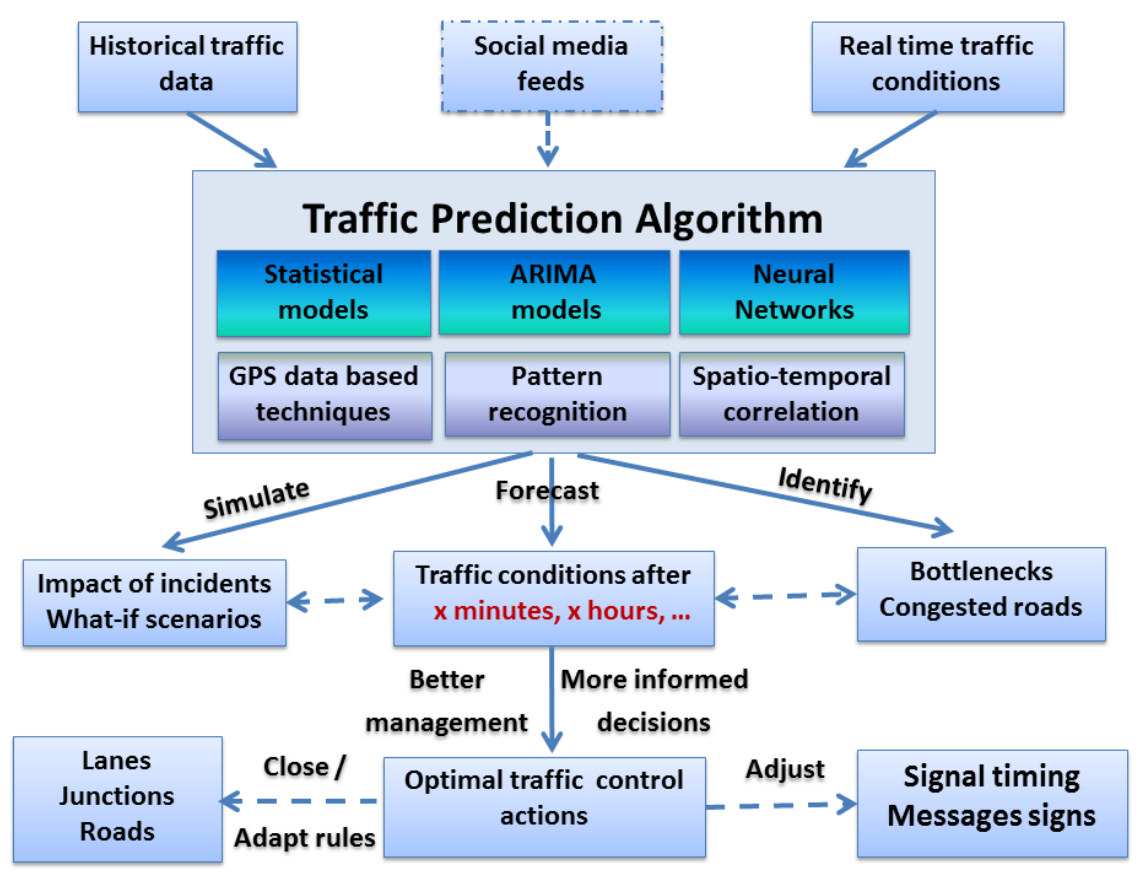

Figure 5: Overview of traffic prediction system and its impact on TMS efficiency

use a combination of simulation, traffic modelling, real-time feeds and historical data to predict how the traffic situation will evolve in the near future. These techniques may also leverage some properties of the road network such as the spatio-temporal correlation for faster inference of traffic jam, as well as other techniques as discussed above. The typical outputs of a prediction algorithm are the traffic forecast and the identification of the bottlenecks. Moreover, it can also explore a set of what-if scenarios through simulation to infer the impact of random incidents on the expected traffic conditions, and therefore more informed decisions will be taken in case of real incident. These decisions may involve adjusting the traffic signal timing, the message signs as well as closing some road lanes or changing the driving rules.

A comprehensive comparison of the major traffic prediction approaches in the literature is provided in Table III. Those approaches are compared based on their achieved prediction accuracy, their scalability level when applied to large scale road networks, the modelling technique used (i.e. parametric or non-parametric), the road environment in which the forecasting approach is applied (i.e. highway or urban area). Moreover, we also considered the type of traffic data source, meaning whether the prediction is based on data collected from fixed monitoring equipment, such as sensors and CCTV cameras, or using mobile data sources such as floating GPS data and SMS, social data feeds etc. This metric is very important as the heterogeneity of data sources and the variety of their format and level of granularity may add extra constraints on the designed prediction algorithm, and may also affect its efficiency and accuracy. Since some prediction techniques impose some constraints on the quality, type and format of the used data feeds in order to ensure high level of accuracy we have also addressed this metric. Finally, the privacy and security concerns that may arise as consequence of the sensitivity of some used data feeds such as social media and GPS data are also covered.

Designing effective tools for fast, scalable and accurate road traffic prediction is a key solution to overcome the weaknesses of the existing TMSs. The fast prediction allows the traffic managers to take early actions to control the traffic load and prevent the congestion state. Fast and accurate road traffic prediction is a paramount technique to enable better efficiency of TMSs and mitigate the awful impact of road traffic congestion. However, most of prediction algorithms are likely to combine historical data with real-time traffic feeds, and apply some advanced and complex modelling approaches to predict the future traffic state, as discussed earlier in this section. Therefore, the legacy simulation approaches are not suitable in this case and distributed simulation is required to allow fast and accurate reaction to the change in traffic congestion in order to mitigate its consequences. The main advantages of fast road traffic simulation are summarized below:

- Enable more accurate recommendations from the TMS to police men regulating traffic at a junction, especially after an incident or during special events. Indeed, after an accident it is a hard task for a human to take the optimal action that mitigates other problems (i.e. accidents, increase the congestion, block other roads etc). Hence, adequate recommendations to traffic authorities, for example in the case of an accident what are the optimal lanes to close to ease traffic congestion?, are needed. This would be based upon exploring the entire solution space (i.e. whatif scenarios) to achieve a reasonably optimal solution. Therefore, this requires extremely fast simulation tools to 
provide the optimal recommendation within a very short time-frame.

- Enable faster and more efficient emergency service delivery (i.e. ambulance, police and fire fighters cars) which significantly reduces the incurred financial loss and save human lives. Here, fast simulation allows the traffic authorities to detect the traffic bottlenecks in advance and take effective actions to prevent them.

- Enable better load balancing of the traffic over the road networks infrastructure, which decreases the traffic congestion and its economic and environmental impact as well as improve road safety.

\section{B. Route planning}

The growing complexity of the big cities' road networks has led to an unprecedented expansion in the automotive navigation systems market. These systems, such as TOMTOM [130] and GARMIN [131], have made the journey of drivers easier and more comfortable due to the valuable information that they provide like the city roads map, GPS localisation and the guided route towards the destination. Despite the popularity of these systems, fast and accurate route search algorithms under the rapid and sudden variation of traffic conditions are still required to accommodate the needs of future smart and autonomous cars. As opposed to static routing algorithms used for shortest path finding in graph theory, route planning algorithms must update the best route assigned to each vehicle as soon as any change in road and traffic conditions that affect at least one road segment that this vehicle should pass through is detected [30], [145], [146].

A typical dynamic route planning algorithm for smart cars is described in Figure 6. This figure emphasizes the main inputs of a dynamic routing algorithm, its output and the road events that may trigger an update of this output. These inputs consist of the city road network modelled as a directed graph in order to reflect one and two ways road segments, the vehicle features (e.g. its height, weight, type ), current traffic conditions and the short term traffic forecasts, as well as the driver preferences. By applying the routing algorithm on the directed graph and taking into account all the other inputs, the best route is returned. This latter should be updated dynamically, during the vehicles journey upon occurrence of any event that may lead to the failure of a road segment included in this route. Notice that the failure of a road segment means its closure due to an incident or road works, or the abnormal increase of travel delay across it. Updating the best route means quickly providing an alternative route that mitigates the detected bottlenecks. One of the challenges here is how to keep the quality of the alternative route very close to that of the failed best route?

Usually, the best route depends on driver preferences which may include one criterion or a combination of several criteria. The travel time is the preferred criterion for most of the drivers due to the critical consequences of the delay. For example, people may lose their job for recurrent late arrival at work, companies may lose money for late delivery of goods to their customers and injured people may lose their lives due to the delay of emergency services. An algorithm that finds the fastest and most reliable route with less computation complexity is, therefore, required. The reliability of the route in this context refers to the probability that no abnormal delay occurs on any link constructing the fastest route during the vehicle journey, as stated in [97].

In addition to the travel time, other criteria such as, the length of the best route, its cost and its associated level of driving easiness and risk are considered by some drivers due to their specific needs. The cost of the route is computed in terms of the fuel consumption level and the number of toll tags included in this route. The fuel consumption is highly dependent on the traffic conditions as well as the road conditions measured in terms of the roughness and the gradient of the road segments of the chosen route [98]. The easiness of driving varies according to the number of turns, number of traffic lights, lanes width and number of hills in the best route, and it could be an interesting criterion for elderly, new drivers and people with poor driving skills. Finally, the level of risk of a route is calculated based on historical statistics about the number and severity of accidents happened on a given route, and some drivers may prefer to avoid this route for safety purposes.

Although several dynamic routing algorithms have been proposed such as, [87] [88], and [89], many problems are still unresolved yet. A noteworthy problem in this context is how can we ensure better usage of the road infrastructure while maintaining a reasonable satisfaction of the drivers preferences? Load balancing mechanisms based on centralized system architecture are more appropriate in this case, but guaranteeing their efficiency is another challenge, especially during the peak hours. We foresee, then, that managing efficiently the growing number of vehicles in smart cities necessitates a mix of centralized and distributed system architectures through leveraging vehicular communication and mobile sensing information during the decision making process. For example, the vehicle can combine the alternative route received from the system with the acquired information from the vehicles ahead to take more information decision about the alternative route that it will follow. Moreover, the system can adapt the quality of the best route assigned to each vehicle according to the level of participation of the driver to mobile sensing process as well as the level of information disclosure. Consequently, this can help to achieve a balance of the traffic load and maintain adaptive satisfaction of the drivers. To get more insight into the proposed approaches in the literature to improve route planning in smart cities, the reader may refer to the following recent papers [43], [44], [45], [46], [47] and [48].

\section{Parking Management Systems}

Another important service that results from data exploitation is parking management which is foreseen to play a key role in improving traffic congestion control and reducing its impact. To be more specific, an advanced parking management system should be operating in tight cooperation with the prediction and routing components of a TMS due to the fact that knowing the volume of traffic heading towards a destination will give more insights about the expected demands on parking spots 
Table I: Comparison of existing traffic prediction approaches

\begin{tabular}{|c|c|c|c|c|c|c|c|}
\hline & Accuracy & $\begin{array}{c}\text { Scalability } \\
\text { level }\end{array}$ & Parametric & $\begin{array}{c}\text { Application } \\
\text { environment }\end{array}$ & $\begin{array}{c}\text { Data source } \\
\text { constraints }\end{array}$ & $\begin{array}{c}\text { Fixed-location } \\
\text { data source }\end{array}$ & $\begin{array}{c}\text { Privacy \& security } \\
\text { issues }\end{array}$ \\
\hline ARIMA & Low & Medium & Yes & Highway & High & Yes & Low \\
\hline Neural Networks & Low & Medium & No & Highway & High & Yes & Low \\
\hline Statistical models & Medium & High & Yes & Urban area & Medium & No & Medium \\
\hline $\begin{array}{c}\text { GPS data } \\
\text { based techniques }\end{array}$ & High & High & No & Urban area & High & No & High \\
\hline
\end{tabular}

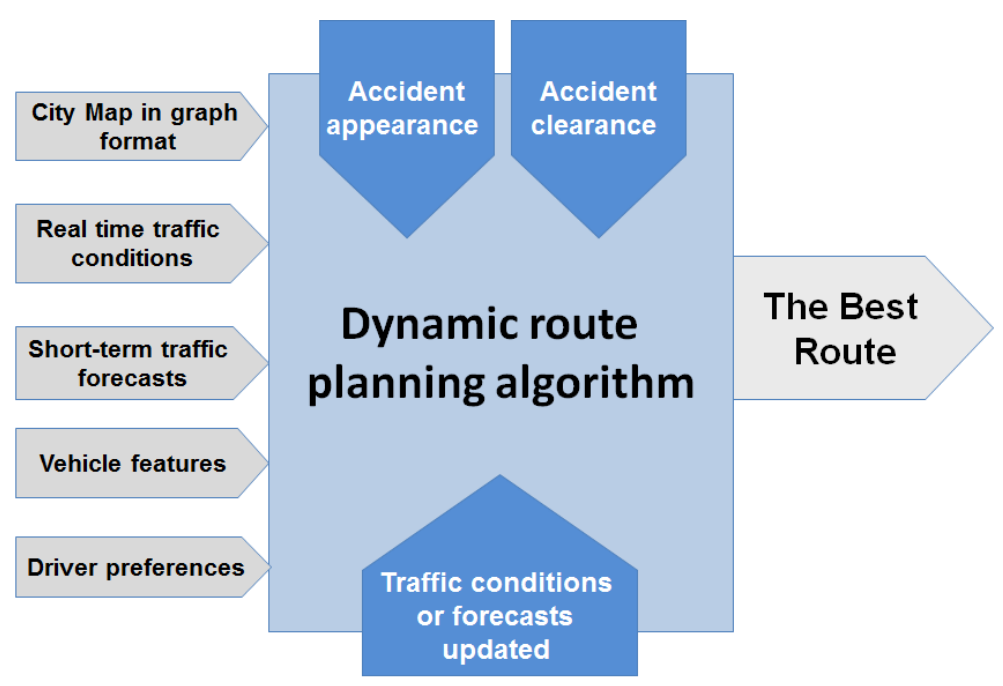

Figure 6: Route planning algorithms: main inputs and functioning

in the near future. Therefore, the routing component may adapt the individual vehicles routes based on its awareness of the available parking spots in an urban area, such that the traffic jam is mitigated and the usage of parking spots is optimized. Figure 7 illustrates a scenario in which the parking management system regularly reports the available parking spots to the routing component in order to increase its awareness of parking availability. Then, the routing component combines this information with the traffic forecasts reported by the prediction component, and adapts the routing decisions accordingly, in order to achieve a global traffic load balance and maximize the usage of available parking spaces. To this end, the routing component may request the parking management system to adjust the number of free spots to be advertised through its mobile applications in accordance with the routing objectives to direct the drivers, for example, towards a specific parking in a given area such that the occurrence of traffic jam is mitigated.

Nowadays, finding an available parking spot is becoming a difficult problem for car drivers. Usually long time is spent looking for available parking places, especially in big cities. Often taking public transportation rather than driving own cars is the preferred option for many people. This problem is mainly due to the lack of efficient parking management systems that ensure early notification of the drivers about the available spots as well as the limited number of available parking spots. The major consequences of this problem are time wasted, increased cost for the journeys and especially, the increase of the congestion level, as the drivers will occupy the limited road infrastructure for longer time than was expected. Therefore, developing efficient solutions for parking management and smart phone based applications (e.g. Park Ya [133] application developed in Ireland and parkinglook [134] in Australia) that signpost parking locations and provide real-time information about spot availability to drivers will certainly alleviate the traffic load on the roads and enhance the TMS effectiveness. In order to contribute to the ongoing efforts aiming at making smart cities happen, worldsensing [135] has developed a green and self-sustainable smart parking solution named Fastprk which makes use of M2M technology to ensure real-time monitoring of available parking spaces. Fastprk has proven its efficiency through the success achieved in the city of Moscow, known by its heavy traffic congestion, where Worldsensing has deployed a huge number of parking monitoring sensors (approximately 15,000) to provide both end users and city council authority with real-time information regarding parking space occupancy. This solution allows the users to find their parking places via electronic street signs or smart phone applications. Fastprk was shown to reduce travel time and fuel consumption, by reducing the time and distance driven to find a parking space. In addition to these applications, other solutions are being investigated by the 
research community such as sophisticated carpooling [136], [137] and public transportation systems that may stimulate the citizens to use these alternative transportation modes, instead of driving their own cars.

Most recently, many researchers have designed solutions to detect available parking spaces and share this information with other cars, via V2V communication, within a specific area. Mathur et al. [49] have focused on urban on street-parking availability and designed a mobile system named ParkNet that uses vehicles equipped with GPS receiver along with ultrasonic sensors to determine the parking spots occupancy while passing by. Based on real data collected in San Francisco, ParkNet has proven an accuracy of more than $90 \%$ in determining the free parking spots. It would also achieve a cost saving of an estimated factor of 10 compared to static sensors deployed at each street-parking place.

Klappenecker et al. [50] have proposed a system to predict the number of available parking spaces when a vehicle reaches the parking. In this system, the parking ticket machine regularly communicates the number of available spaces to the vehicles upon arrival using Markov chain based estimation. This system, however, doesn't exploit the free spaces efficiently as more than one vehicle may drive to the same parking spot as described in [53]. To overcome this drawback, [53] proposes a reservation protocol that allows a vehicle to claim a spot when it becomes free, thus an optimal use of the available spots is guaranteed in this case.

Panayappan et al. [51] have proposed to deploy sensors on the sides of each vehicle to detect the presence of any vehicle in the place next to it. This is a useful mechanism to prevent abuse as the multiple cars and car park sensors will check whether the space is free. In the paper by Kokolaki et al. [52], each vehicle gathers the location of each empty parking space and then forwards this over the ad-hoc network. This approach was compared with a non-assisted search and centralized server approach. The VANETs based scheme didn't always outperform the centralized server but the paper highlights the fact that the VANETs based scheme requires no additional infrastructure to be built, so it is a much more cost effective solution.

A decentralized and scalable parking spots information system has been developed in [55] to inform the drivers about parking spots availability in an urban area. This system makes use of VANETs to disseminate micro and macro parking information either locally or at large scale, respectively. Micro information refers to free parking spots coordinated by one automat while macro (i.e. aggregated) information covers several parking within one urban area. This system has shown high efficiency under realistic model of German city in which $5 \%$ of the vehicles, out of 10000 , are equipped with wireless communication capabilities. To complement the previous work, Caliskan et al. [54] have developed a model using homogenous Markov chains and queueing theory that estimates the future occupancy of parking spots located within the vehicle's destination area at its arrival time. Based on the parking information received through VANETs the vehicles apply this model to decide about its orientation to one of the available parking.
Szczurek et al. [56] have proposed a machine learning algorithm for determining whether a given car park will have a space to park. In this system, when a vehicle leaves a parking space, it sends a message over VANETs announcing that a parking space has become available and specifies its corresponding coordinates. This work has shown a reduction in the time spent searching for a car park space of over $25 \%$ compared to a blind search.

It is well known that on street parking offers most car parking spaces in cities, which means that an efficient management of these spaces may lead to a substantial benefits for both city and citizens. Unlike off-street parking lots where the car park gate can be used as a sensor to assess the occupancy level, a sensor per parking space is required to monitor and detect the availability of on street parking, which represents a significant cost for their deployment. However, to reduce this cost, Evenepoel et al.[57] have proposed to deploy the sensors on a fraction of on-street car park spaces only and then use extrapolation to infer the amount of cars parked in the entire city. A probabilistic model was devised to quantify the reliability and efficiency of the proposed approach and the obtained results were promising, as they show that ensuring slightly less than $2 \%$ of parking space coverage by sensors would be optimal. Therefore, a significant reduction of the sensor deployment cost would be achieved. However, the main shortcoming of using so few sensors is that some drivers might be tempted to "cheat" in order to guarantee easy and fast parking for themselves or their colleagues at work. For example, an employee may intentionally park on the parking spot equipped with a sensor so that the road would appear full to other users, whereas this is not the case.

\section{Vehicular Networking Support For Data GATHERING AND SERVICE DELIVERY}

In this section, we explore the different routing approaches used in vehicular networks to disseminate the gathered data among the vehicles as well as the information transmitted by the TMS or other service providers towards all the vehicles or a sub-set of them, discuss their advantages and disadvantages, and provide a comprehensive comparison of their main features. We also address the challenging problem of beacon congestion control in IEEE 802.11p MAC layer and briefly describe the pioneer works that have dealt with this issue. Moreover, we outline the recent advances in simulation tools for road traffic and VANETs based applications, and highlight their main features and degree of realism.

\section{A. Vehicular Ad-hoc Networks (VANETs)}

Vehicle-to-Vehicle (V2V) and Vehicle-to-Infrastructure (V2I) communications are expected to play a key role in the development of TMS in smart cities. The efficiency of this type of communication depends on the reliability of the WAVE system and mainly on IEEE 802.11p MAC protocol [40], in addition to the information dissemination (i.e. routing) protocols. In this section, we will present, classify and compare the most significant protocols proposed to find the best route for the exchanged packets among non-neighboring vehicles 


\section{Parking Apps}
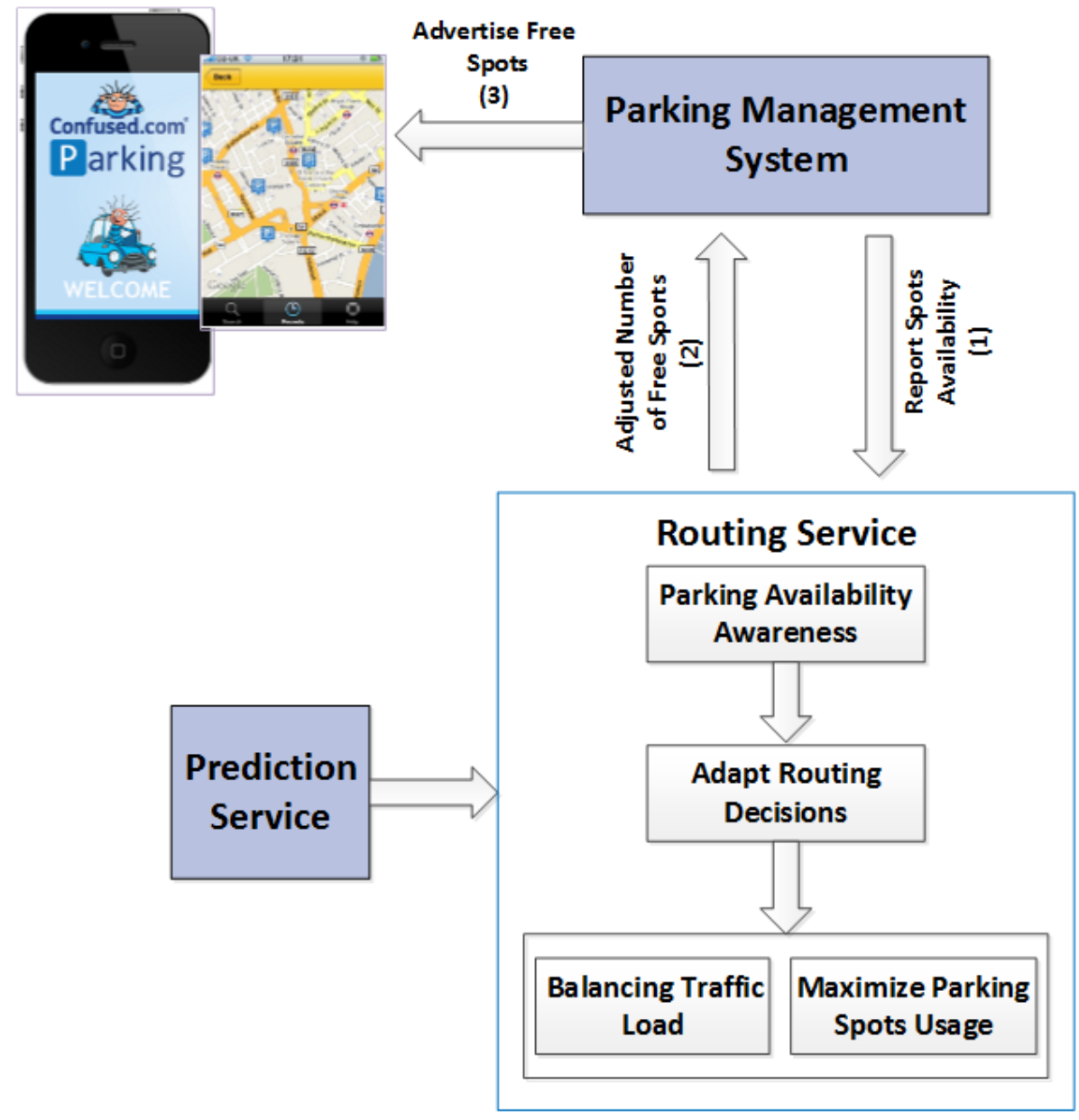

Figure 7: Illustration of Prediction, Routing and Parking services cooperation

in road network. Usually, these protocols use either the road network map, the vehicles mobility model, both of them or none of them to accurately determine an end to end connected route between the source and destination vehicles. Therefore, we classify these protocols, as shown in Figure 8, into four categories based on their awareness of these two parameters (i.e. the map and mobility model), as described below.

1) Context-unaware routing protocols: these routing mechanisms do not take into account the road map and nor the predicted mobility of vehicles.

Greedy Perimeter Coordinator Routing (GPCR) [3] is a position based routing protocol. It uses the fact that streets and junctions form a natural planar graph. In this protocol, messages are forwarded along the street with decisions only taken at junctions. GPCR uses a repair strategy to get rid of local minimums. This protocol does not require a static streets map as it can heuristically detect the junctions on the road, however it is not resilient to network partitioning that may occur due to links loss.

In the 3rule routing protocol [86], a set of sink nodes aware of their location are deployed and configured to form the network infrastructure. Other nodes then set up a virtual location by judging their distance from those sink nodes. For efficient routing, a greedy geographic approach is used to route the exchanged messages among the nodes, and the evaluation results have shown that this scheme outperforms GPSR (Greedy perimeter Stateless Routing) in terms of energyefficiency, path length and robustness.

In Location-Based Routing Algorithm with Cluster-Based Flooding (LORA_CBF) [4], the cluster based flooding mechanism is used, where a number of gateways are chosen for inter clusters communication, in addition to the cluster head. When a data packet needs to be sent, the sender first checks its routing table to find the location of the destination node. If this location is missing then a location request message is broadcasted to the network. Upon reception of this request, the destination node or any intermediate node, which has fresh location information of the destination node, sends a location reply message to the source. The data packet is then transmitted through this route. The hierarchical architecture of LORA_CBF leads to shorter route discovery time but the overhead increases considerably [41]. 


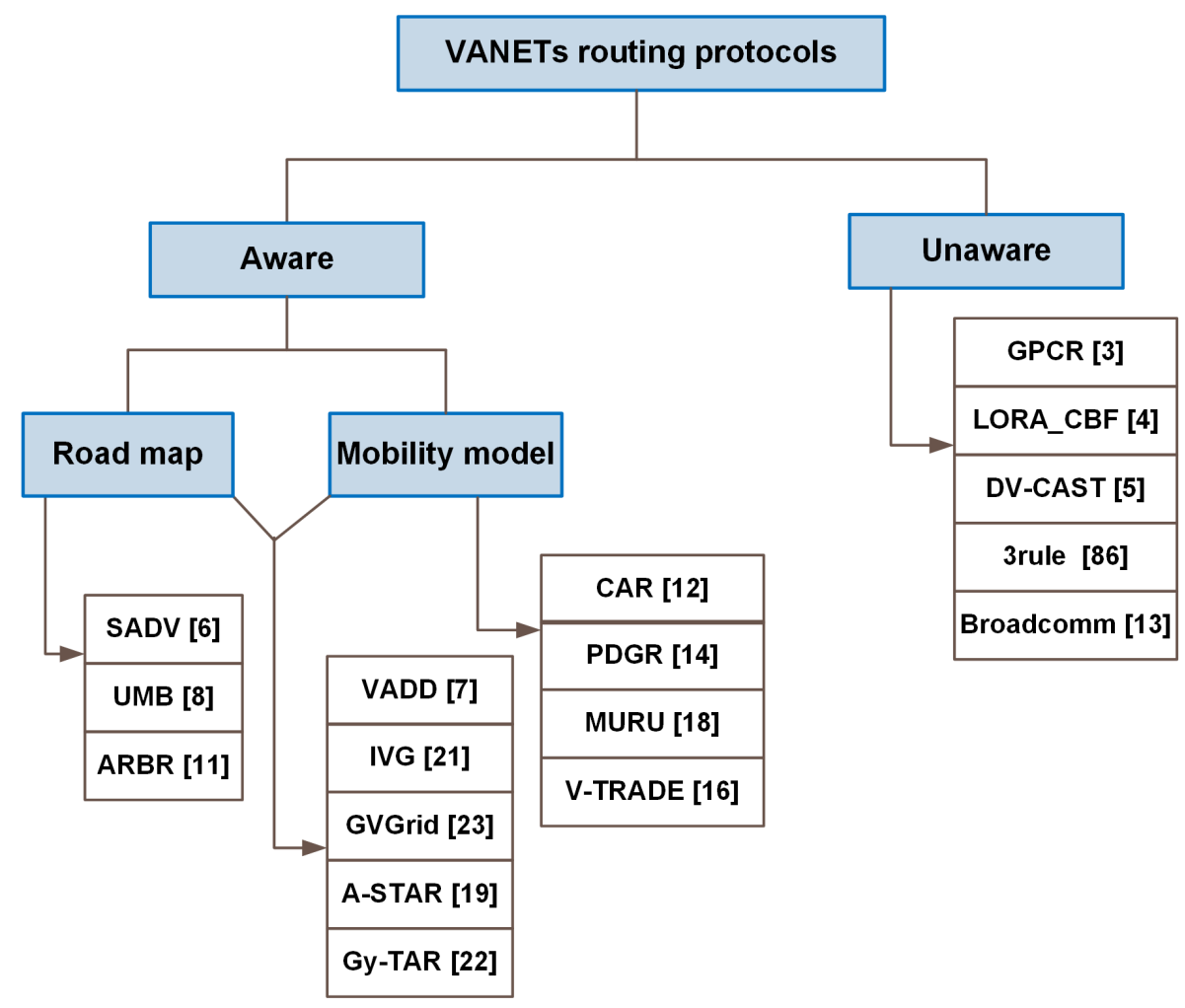

Figure 8: A classification of VANETs routing protocols based on their awareness of the mobility model and the road map

DV-CAST [5] applies two different approaches according to the network connectivity, it uses the broadcast suppression technique in order to reduce the broadcast overhead in case of a dense network, while a store-carry and forward method is used in a sparse network. The network density level is determined based on the size of one-hop neighbors list. This protocol overcomes some of the previous protocols' limitations as it reduces the broadcast storm and adapts its routing approach to deal with network disconnection problem.

Broadcomm [13] is a fast routing protocol specifically designed for safety applications. It divides the highway into virtual cells which move along at the average highway speed. At the center of each virtual cell, some nodes are designated as cell reflectors which, in turn, act as virtual base stations. It is worth mentioning that cell reflectors are similar to cluster heads, except that several cell reflectors may co-exist within one cell. The main weakness of this protocol is the high incurred overhead.

2) Map-aware routing protocols: in this category of routing protocols, map information is a cornerstone for calculating the end to end path for a data packet.

SADV [6] is an infrastructure based routing protocol that presumes the existence of a static node (i.e. a Road-side Unit (RSU)) at each junction. Each RSU has a digital street map to determine which road presents the best trajectory. A data packet waits at the RSU till a route to the next intersection is established. This route is selected based on the delay estimation of each road in order to achieve a near optimal choice. SADV improves the packet delivery ratio and presents an enabler for RSUs placement in road networks.
Other protocols in this category are Urban Multi-Hop Broadcast protocol(UMB) [8] which addresses the broadcast storm and hidden nodes problem and ARBR [11] which uses carry and forward scheme to overcome network fragmentation issue.

3) Mobility model-aware routing protocols: the following routing mechanisms leverage the knowledge of vehicles' mobility models for messages routing purposes.

Connectivity-Aware Routing (CAR) [12] uses a greedy forwarding approach with anchor points to find the route relaying origin-destination pairs. In CAR, the messages are forwarded to the closest node to the next anchor point instead of the closest node to the destination, and the location of this latter is tracked so that the route can be adjusted to provide connectivity even if the destination has moved a great deal. Notice that the incorporation of CAR routing approach in GPSR has shown an improvement of the performance by $30 \%$, however the main shortcoming of this protocol is its inefficiency to handle different sub-paths under frequent topology changes.

Predictive Directional Greedy Routing protocol (PDGR) [14] routes the vehicles based on both their current and predicted positions. It applies a greedy strategy and forwards the messages in the direction of the destination vehicle without a predetermined route. PDGR considers both the position and movement of a vehicle for forwarding decisions. It has been shown that PDGR outperforms GPSR in terms of delay, delivery ratio and overhead.

MUlti-hop Routing for Urban VANET (MURU) [18] is another protocol that attempts to increase packet delivery ratio 
through early detection of the broken links in the VANETs, whereas Vector-based TRAcking DEtection (V-TRADE) [16] uses vehicles positions and directions to ensure more efficient routing.

4) Map and Mobility mode-aware routing protocols: This class of protocols exploits both the road map and vehicles mobility model to ensure a robust route for messages delivery among vehicles.

VADD (Vehicle-Assisted Data Delivery) [7] is designed specifically to route data packets in sparse VANETs with frequent network fragmentation due to the high mobility of vehicles. These packets will be transmitted over the routes with shortest transmission delay. In case of network fragmentation, the packet is forwarded to a vehicle that crosses the network partitions first and then forwards it towards the destination. VADD determines whether there is a direct route to the destination by analyzing the map of the area and the traffic conditions around it. This protocol ensures higher delivery ratio compared with GPSR [17] and DSR [2], as stated in [7]. However, a large delay may occur under varying topologies and vehicles density.

Inter-Vehicles Geocast (IVG) [21] is a safety based protocol that broadcasts an alarm message to all the vehicles in a given area if there is a danger. The vehicles are in the danger area if this danger is in front of them. In this case, these vehicles constitute the multicast group that will receive the alarm message, then forward it in the backward direction. In order to reduce the gratuitous alarm messages IVG takes into account the braking distance before broadcasting this message. However, if the danger is immediate the alarm message is sent, regardless of this distance, to prevent crashes.

GVGrid [23] uses a reactive routing approach to construct a route from a fixed source node to another vehicle located within a specific geographic area. GVGrid divides the road network map into a set of uniform squares and assume that each vehicle is equipped with a digital map and is aware of its location and direction through GPS. This protocol ensures route recovery in case of link break due to vehicles mobility. GVGrid uses stop signs and highways with constant vehicles distance as prediction indicators for vehicles mobility to enhance messages routing.

In addition to the above protocols, A-STAR [19] and GyTAR [22] have been also proposed in this category. A-STAR is an Anchor-based Street and Traffic Aware routing protocol that combines street map and bus routes information to determine the fastest path that exhibits higher connectivity. A-stars use of the right hand rule is inefficiently biased in one direction, as stated in [20]. The latter protocol, GyTAR, employs a greedy strategy that takes into account real-time traffic conditions and road topology, in addition to a recovery strategy to overcome local optimum.

To summarize, each of these routing protocol categories has advantages and shortcomings and might be suitable for some road traffic scenarios and not applicable in others. Moreover, the awareness of vehicle mobility models and road network maps might not be sufficient to meet the requirements of safety applications, especially in emergency scenarios where both fast and reliable dissemination of danger alerts are compulsory.
Therefore, an ideal routing protocol for VANETs in the context of smart cities should be aware of extra parameters, in addition to the mobility model and map, such as the shape of the roads, destination of other vehicles, channel interference level, etc.

In the Table II, we compare the different routing approaches presented above according to the following criteria: the incurred communication and computation overhead, to what extent the protocol is scalable?, the end-to-end delay of the transmitted packets, its efficiency in terms of packet delivery ratio, its resiliency to VANET fragmentation due to the high mobility of vehicles, whether its applicable in urban or highway scenarios or both of them, and finally whether it requires the help of the road-side infrastructure or not?.

In addition to the routing function, the media service in VANETs has become a hot topic in recent years and several contributions have been proposed to enhance the efficiency of VANET applications and services. In [140], the authors have addressed VANET-based entertainment services such as video streaming, file sharing, mobile office and gaming etc. As noted in the paper, these services can make the drivers and passengers travel experience more pleasant, however a number of research challenges need to be overcome to make those services efficient and robust. To this end, several challenges have been highlighted such as frequent network disconnection, high mobility of vehicles etc. the authors discuss also the requirements that the existing channel access and resources management schemes in VANETs need to satisfy in order to be suitable to support entertainment and safety applications. Another work [141] has focused on Video on Demand (VoD) services provided to vehicles using P2P networks. This work proposes Quality oriented User centric VoD (QUVoD) designed specifically for vehicular networks. QUVoD introduces a new grouping based storage strategy as well as a novel speculation-based prefetching strategy. The simulation results of this work have proven its superior performance benefit in comparison with the state of art solutions.

\section{B. IEEE 802.11p congestion control}

Congestion control is probably the most challenging issue at MAC layer in VANETs [79] given the fact that IEEE 802.11 is well known by its scalability problem. The research community has highlighted the importance of congestion control in VANETs, and the ETSI ITS framework [80], [81] has defined a set of mechanisms to deal with this issue such as data rate adaptation, transmission power control and beacons frequency adjustment. However, these mechanisms are still inefficient due to the characteristics of the control channel (CCH) [40] in vehicular environment as well as the large number of vehicles unusually contend for channel access.

Most of the proposed solutions to control the congestion in VANETs focus on adjusting the transmission power [35], [82] used for broadcasting the beacons to prevent the congestion state or at least reduce its impact on the performance. However, in some situations, this may cause an isolation of some vehicles when vehicles density decreases. This is because of the highly fluctuating topology of VANETs as the vehicles move very fast and change their directions often. To take into 
account this specific feature of VANETs, [83] has proposed to assign data rates based on the average utility of the messages transmitted by each vehicle. Thereby, vehicles transmitting information with a high utility (e.g. safety messages) for the VANET are allowed to consume a larger part of the available bandwidth. This scheme requires that the vehicles share the information that allows to each of them to calculate its own data rate. Hence, the overhead incurred by the exchanged messages may significantly reduce the available bandwidth, especially when the number of vehicles gets larger.

Data rate control [84] has been also proposed to deal with MAC layer congestion in the ETSI ITS framework. The idea behind this adaptive mechanism is that a higher data rate implies the message occupies the channel for a smaller duration, thus allowing more transmissions to take place. An important observation here is that the higher the data rate provided by a modulation, the higher the signal-tointerference ratio (SIR) required at the receiver side in order to correctly decode the message. Simulation studies (e.g. [84]) have shown that the reception probability for geographically close vehicles is hardly affected and, in these conditions, adjusting the data rate gives similar results with transmission power control. Therefore, choosing the modulation based on the local vehicles density seems to be a promising solution in crowded environments like VANETs.

Other works, such as [85] and [29], have proposed to increase the bandwidth available for the $\mathrm{CCH}$ to reduce/mitigate the impact of congestion. In [29], the authors have proposed a cognitive radio technology based technique that allows the vehicles to opportunistically use the detected holes in the primary users frequency spectrum in their neighbourhood. This extra bandwidth gained by the vehicles could be mainly used to ensure rapid transmission of alert messages in emergency cases. Radio cognitive technology has been also applied in the following works [37], [38], [42] and [39] to improve the reliability of safety applications in VANETs. In addition to the above discussed works, the reader may refer to [27], [25], [26], [34], [24], [24], and [36] to get a broader idea about the different solutions designed to mitigate the congestion problem in VANETs.

\section{Current trends in road traffic and VANETs simulation}

In June 2013, researchers from the Transportation Research Institute of University of Michigan have showcased V2V and V2I communication demo [132], during which the vehicles equipped with IEEE $802.11 \mathrm{p}$ communication technology were able to exchange their position, speed and direction with similarly equipped peers as well as with the roadside infrastructure like traffic lights and tollbooths. This unprecedented real world vehicular communication experiment has involved 2800 vehicles of different types and shown that vehicular communication technology can play a key role for improving roads safety. Despite that, simulation remains one of the strategic tools for evaluating the performance of the developed VANETs communication protocols and ITS applications due to the inaccessibility or the high cost of the resources needed (e.g. vehicles equipped with communication capabilities, roadside units etc) to carry out real world tests. Although many discreet-event network simulators, such as ns-2, ns-3, OPNET, OMNet++ and QualNet, have been widely used by the researchers to validate their ideas and approaches, they cannot be used in ITS scenarios without an accurate vehicular mobility model. Moreover, if the ITS application influences the behaviour/mobility of the vehicles then a real-time bidirectional coupling of network and road traffic simulators is required [78]. To this end, some European research projects have recently developed platforms integrating both network and microscopic traffic simulators to improve the accuracy and realism of ITS solutions evaluation. The most known platforms are Veins [93] which is based on OMNeT++ and SUMO, and iTETRIS [91] that integrates NS-3 with SUMO.

There are two major avenues for road traffic and IVC modeling and simulation. One approach, taken by macro simulators, considers the overall traffic flow modeling and simulation on the road network, and no detailed level information and related input, output or processing (e.g. at vehicle level) are being considered. The second avenue is taken by micro simulators, which simulate individual vehicles in the traffic systems. Vehicles are seen as important actors in the road network system and not only are mobile in this context, but also generate, process and sink network data traffic. In order to best address the current research and development needs, this section focuses on micro traffic simulators. A study conducted between 2009 and 2011 on top level international conference papers [92] has identified the three most popular road traffic micro simulators used by the research and development community. The Simulation of Urban Mobility (SUMO) has been reported as used by more than $20 \%$ of the papers with a peak of $30 \%$ in 2010. The use of SUMO is almost constant, trend which continues today. In contrast, the dedicated vehicular network movement simulator VanetMobiSim, which has been used in nearly $20 \%$ of the publications surveyed in 2009, has experienced a marginal use lately. VISSIM, which is a commercial tool, maintained an average proportion of about $6 \%$ during last three years. In meanwhile other micro traffic simulators have also been proposed and are being used by the research and development community focusing on vehicular traffic modeling and simulations. Next most important of these solutions are presented.

In what follows, we will briefly discuss the most used road traffic simulation tools and applications in the research community, and highlight their main features and limitations, as shown in Table III.

1) SUMO: SUMO is an open-source traffic micro-simulator designed to handle large road networks. Sumo was mainly developed by the Institute of Transport Systems at the German Aerospace Center. SUMO allows for space-continuous and time-discrete vehicle movement modeling and simulations. Some of the features include: different vehicle types, multilane streets with lane changing support, different right-of-way rules, traffic lights, etc. SUMO provides network-wide, edgebased, vehicle-based, and detector-based outputs. It has a fast openGL graphical user interface, scales very well (i.e. tens of thousands of streets) and provides fast execution speed (e.g. 100.000 vehicle updates/s on a $1 \mathrm{GHz}$ machine). It also supports interoperability with other applications at run-time. 
Table II: Comparison of the main characteristics of the surveyed vehicular routing protocols

\begin{tabular}{|c|c|c|c|c|c|c|c|c|}
\hline & \multicolumn{8}{|c|}{ Characteristics } \\
\hline & $\begin{array}{c}\text { Communication } \\
\text { overhead }\end{array}$ & $\begin{array}{c}\text { Computation } \\
\text { overhead }\end{array}$ & $\begin{array}{c}\text { Scalability } \\
\text { level }\end{array}$ & Latency & $\begin{array}{c}\text { Delivery } \\
\text { ratio }\end{array}$ & $\begin{array}{c}\text { Network partitioning } \\
\text { resiliency }\end{array}$ & $\begin{array}{c}\text { Target } \\
\text { scenario }\end{array}$ & $\begin{array}{c}\text { Infrastructure } \\
\text { dependant }\end{array}$ \\
\hline GPCR [3] & Low & Low & Medium & High & Low & No & Urban & No \\
\hline 3rule [86] & Low & Low & Unknown & Unknown & High & No & All & Yes \\
\hline LORA-CBF [4] & Medium & Low & High & Low & High & Medium & Urban & No \\
\hline DV-CAST [5] & Low & Low & High & Low & Medium & Very High & All & No \\
\hline SADV [6] & Low & Low & Medium & Medium & Medium & High & Urban & Yes \\
\hline VADD [7] & Low & Medium & Medium & Medium & Low & High & Rural & No \\
\hline UMB [8] & Medium & Medium & Medium & High & Medium & Medium & Urban & Yes \\
\hline ARBR [11] & Low & Medium & Medium & Medium & High & High & Urban & Yes \\
\hline CAR [12] & Medium & Medium & Medium & Medium & Medium & Medium & All & Yes \\
\hline BROADCOMM [13] & High & Low & Medium & Low & Low & Medium & Highway & Yes \\
\hline V-TRADE [16] & Medium & Low & Medium & Medium & Low & No & Highway & No \\
\hline PDGR [14] & Medium & Medium & Medium & Medium & Medium & No & Urban & No \\
\hline MURU [18] & Low & Medium & Medium & Low & Medium & High & Urban & No \\
\hline A-star [19] & Medium & Low & Medium & Medium & Low & Medium & Urban & No \\
\hline IVG [21] & Low & Low & High & Low & Medium & High & Highway & No \\
\hline GyTAR [22] & Low & Low & Medium & Low & Medium & High & Urban & No \\
\hline GVGrid [23] & Medium & Medium & Medium & Medium & Medium & Medium & Urban & Yes \\
\hline
\end{tabular}

Sumo allows the user to import different sources such as VISSIM and open street map. Sumo is coded in C++ [90].

2) iTETRIS: iTETRIS has opted for integrating two wellknown and widely used open source simulation platforms. SUMO (http://sumo.sourceforge.net) as an open-source microscopic traffic platform and Network Simulator 3 - NS3 (http://www.nsnam.org/) for wireless communications modeling and simulations. The capability to perform large-scale simulations and to support multi-radio/technology nodes was a key-parameter for the selection. iTETRIS resulted to have the best performance in terms of scalability [65].

3) STRAW: STRAW (STreet RAndom Waypoint) is an open-source traffic simulator built by researchers at the AquaLab at the Northwestern University, US. STRAW runs on top of the highly efficient JiST/SWANS discrete-event network simulator. STRAW includes a realistic mobility model with very good level of details for vehicular networking research. STRAW street topology modeling uses real life TIGER street maps collected by the US Census Bureau. It includes streets whose structure allows for identification of segments, ramps, intersections, etc. STRAW models vehicular node movement including acceleration, deceleration, etc. The mobility model addresses aspects such as vehicular congestion and traffic control by deploying specialised mechanisms to impose infrastructure limitations on the traffic flow [94].

4) VISSIM: VISSIM is a microscopic, behaviour-based discrete event traffic simulation system modeling motorway and urban road traffic. Based on complex mathematical models, the position of vehicles is calculated and updated regularly. VISSIM offers a high level of complexity in terms of display with both 2D and 3D views. VISSIM can be used to investigate private and public transport including in particular pedestrian movements scenarios. The use of VISSIM is moderate with roughly 6,000 individual PTV Vissim Licenses around the world. There has also been roughly 3,800 downloads of the VISSIM demo in 2012 [95].

Additionally there are several applications which allow for vehicular traffic modeling, simulations and analysis. Among these for instance SIDRA TRIP allows to compare travel conditions on alternative routes, to assess network traffic performance, and to analyse vehicle movements and traffic performance. It is based on collected GPS data inputted by the user to form traffic traces, which are then used during simulation and analysis. SIDRA has quite a small user base compared with SUMO and VISSIM [96].

\section{SMART VEHICLES AND TMS INTERACTION}

Vehicular communication can play an essential role in improving the efficiency of both data collection and TMS reaction to some circumstances or emergency events. Smart vehicles are usually equipped with on board sensors that are able to detect both in-vehicle events as well as the surrounding traffic conditions. These inner events such as sudden deceleration and airbag tripping are immediately reported to the neighbouring vehicles and the Road-Side Units (RSUs). On 
Table III: A summary of the main features and limitations of road traffic microscopic simulation tools and applications

\begin{tabular}{|c|c|c|c|c|c|}
\hline & VANETMobisim & STRAW & SUMO & PTV Vissim & SIDRA TRIP \\
\hline Transport Modes & Truck \& Cars & Cars & Multi-modal & Multi-modal & Cars \\
\hline Accuracy & Time step of $1 \mathrm{~ms}$ & & Time step of $1 \mathrm{~s}$ & Time step of $0.1 \mathrm{~s}$ & Time step of $1 \mathrm{~s}$ \\
\hline Scalability & Medium & Medium & $\begin{array}{l}\text { High } \\
\text { Up to } 100,000 \text { vehicles }\end{array}$ & $\begin{array}{l}\text { Very High } \\
\text { No-built in limits }\end{array}$ & $\begin{array}{l}\text { Very low } \\
\text { Single car }\end{array}$ \\
\hline GUI & Limited & Limited & $2 \mathrm{D}$ view & 2D\&3D view & $2 \mathrm{D}$ view \\
\hline $\begin{array}{l}\text { Realistic simulation of } \\
\text { pedestrian and passengers behaviors }\end{array}$ & No & No & No & Yes & No \\
\hline Parking management & No & No & No & Yes & No \\
\hline C2X support & No & No & No & Yes & No \\
\hline $\begin{array}{l}\text { Accurate analysis of } \\
\text { single cars trip }\end{array}$ & No & No & No & No & Yes \\
\hline Popularity & Medium & Low & Very high & Medium & Low \\
\hline Licence needed & No & No & No & Yes & Yes \\
\hline
\end{tabular}

the other hand, the received events from other vehicles or road sensors are processed and reported similarly to the inner ones. The gathered traffic data from smart vehicles are then analyzed and combined with other data feeds in order to speed up traffic congestion detection and improve the congestion levels evaluation accuracy. In this context, these data need to be quickly disseminated with high transmission reliability, especially if it reports safety critical events. Thus, appropriate dissemination protocols are required. In what follows, we discuss a set of scenarios in which the interaction between the TMS and smart vehicles will significantly help to reduce traffic congestion and improve roads safety.

In the first scenario, we propose to investigate the possibility of affecting/changing the cars behavior (e.g. speed, optimal route etc) and the driving policies (e.g. maximum speed, minimum speed, reserved lanes etc) rather than only closing some road segments as proposed in the legacy systems. In this case, the cars need fast and accurate coordination when they change lanes to temporarily use a lane which was reserved for buses or slow cars, in order to prevent crashes. To this end, a real-time dissemination of lane change notification is a must since lane change in this context may lead to collision if more than one car move to the same lane simultaneously and without coordination. Moreover, the road-side infrastructure may also make use of the information exchanged between the vehicles through the transmission of beacon messages. It will then combine the content of these beacons (i.e. vehicle speed, position, heading etc) with the reported data from the road monitoring equipment, as shown in Figure 1, to speed up the congestion detection and improve its accuracy, and thus the TMSs can take early actions to control the traffic congestion.

In the second scenario, we propose that the road-side infrastructure (e.g. traffic light controller at an intersection) communicates the remaining time for the current traffic light cycle (i.e. to switch from green to red and vice versa) to the approaching vehicles, in order to reduce their waiting time when they reach the intersection. In this case, the vehicles are informed about the optimal speed which allows them to cross the intersection without stopping. To achieve this goal, the vehicles need to coordinate between each other to adjust their current speed according to the speed advised by the infrastructure. The purpose of the coordination between the vehicles is to avoid collision when they adapt their speed according to the information received from the traffic light controller.

One of the most critical consequences of traffic congestion is the delay of emergency services, such as police intervention, fire and rescue operations as well as medical services. This scenario aims to reduce the latency of emergency services delivery by dynamically adjusting traffic lights, changing related driving policies, recommending behaviour change to drivers, and applying essential security controls [28]. This will create green route for these vehicles and significantly reduce their response time, which may save human lives and reduce the induced damage/loss in case of fire or robbery. The TMS should be also able to control the behaviour of nonemergency vehicles to ensure minimum number (ideally zero) of crashes, minimum disruption to the regular traffic flow, and satisfaction of security requirements to prevent any misuse of the system. To make this scenario viable and valuable in real road environment, some specific actions should be taken by both TMS and smart vehicles in addition to some requirements which should be satisfied, such as:

- The traffic light controller is made aware of the approach of an emergency car through a special message sent by this car towards the infrastructure when it approaches the intersection. Alternatively, if an induction loop system is in place we can imagine that those cars are equipped with a special tag (hardware) to distinguish them from the other cars. Hence, whenever an emergency car passes 
through the induction loop system this latter automatically generates a special message to request the traffic light controller to switch to green or to extend the green light cycle till the emergency car crosses the intersection.

- Fast and reliable V2V and V2I communication protocols are needed to enable real-time interactions between the TMS and the smart vehicles.

- Adequate security mechanisms should be added to this system to prevent its misuse by malicious cars that may spoof the identity of an emergency car for different purposes.

- The TMS should apply advanced decision-making solutions to find alternative routes to divert the normal vehicles from the dangerous area in order to protect the drivers lives and ease the access for emergency vehicles. These solutions must consider the real-time contextual factors as well as the security requirements.

An example of an adaptive TMS in emergency scenario is depicted in Figure 9, in which the Local Traffic Controller (TLC) uses the gathered information about the traffic conditions to clear the way for the ambulance. This can be achieved by changing the traffic light cycles, and defining new driving policies and announcing them to the cars through the set of RSUs deployed along the roadside.

\section{SECURIty Threats Against TMS}

Secure and highly efficient TMSs, which are responsible for critical operations such as transportation infrastructures supervision and road traffic control, are essential to strengthen the national security of any country in the world and support its economic expansion since both governmental and private companies rely on these infrastructures to successfully accomplish their daily operations. However, both TMSs and road infrastructures are vulnerable to a bunch of threats that range from environmental and accidental events to malicious attacks, and may lead to sustained outages and wide disruption. Advanced TMSs and Traveler Information Systems (TISs) exploit the technologies used by transportation infrastructures to enable real-time collection and dissemination of information about traffic flow conditions and transit schedules, in order to decrease the congestion level and traffic incidents. Additionally, TMSs may provide other services for public transit systems, commercial vehicle systems as well as emergency management systems. Any disruption of these services can lead to destructive impact on public safety and/or national economy depending on the target system. These disruptions can be caused by hackers, terrorists, foreign enemies, or unauthorized users, and can be a consequence of power failure, natural disaster like a storm or tornado, or a telecommunication outage.

Despite the efforts of road and public authorities to reinforce transportation systems security, they are still prone to numerous threats that may target the critical road infrastructure including the monitoring equipment, the connected smart vehicles system, the smartphones based ITS applications, or the core of the TMS in order to bring it to halt. These threats become more serious with the recent trends on integrating advanced technologies for road traffic surveillance and management, leading to an increasing number of vulnerabilities at several levels. For example, the use of wireless sensors for data sensing and gathering may lead to several attacks inherited from WSNs technology and wireless multihop communication paradigm, which affects both the integrity and quality of the collected traffic data. Moreover, leveraging smart vehicles for spreading warning notifications about onroads emergency events may lead to severe consequences that range from increasing traffic jams to economic damages and human lives loss in case of robbery or terrorist attacks. For example, a vehicle advertising an accident in a given road segment not covered by visual monitoring equipment may succeed to divert the traffic from this particular area in order to undertake a criminal act or just create traffic jam in the surrounding. Indeed, road transportation networks are very attractive targets for criminals aiming to inflict big loss to the city and road authority, serious panic among population and create spectacular media images, as those networks are usually used by large numbers of cars (drivers) at predictable times in predictable places, especially in big cities.

Furthermore, traffic-aware or context-aware content security has recently attracted a lot of attention from the research community and several issues have been identified in this regard. In [142], the authors revealed that VANETs can be an easy target of indirect attacks through exploiting the sensors deployed for traffic information collection and reporting. A malicious user can, in this case, remove/drop certain sensor readings indicating traffic congestion in a given area, or spoof the identity of some road traffic monitoring sensors and insert fake values indicating a traffic jam in road segments with low traffic, which may mislead other vehicles as well as traffic controllers and lead to devastating consequences. Besides these security threats, Sybil attack is another type of attacks very hard to detect especially in such highly dynamic environment like VANETs. To cope with it, data-centric misbehaviour detection schemes have been applied. The two main mechanisms used are consistency check and plausibility check. The former mechanism checks the consistency of traffic information reported by vehicles in the same area and finds out any deviating values reported by either malicious or faulty nodes. The latter mechanism usually has a model of the real world used to check whether the reported values comply with this model and thus detect any unrealistic values advertised by misbehaving vehicles. In addition to the above schemes, [143] has proposed novel scheme to deal with malicious nodes attempting to spread forged messages in VANETs for both safety and non-safety applications. To this end, the bilinear pairing has been used to ensure fast and accurate verification of the authenticity of the messages content. This scheme assumes that each vehicle is equipped with a tamper-proof device. The obtained results have proven its effectiveness and supremacy over the existing solutions in the literature.

As discussed above, TMS is vulnerable to numerous security attacks that exploit the vulnerabilities of the equipment and technologies involved in its operations. These attacks can be categorized into three main categories according to the target entity. The first category concerns the attacks targeting the critical road infrastructure through an unauthorized access to or 


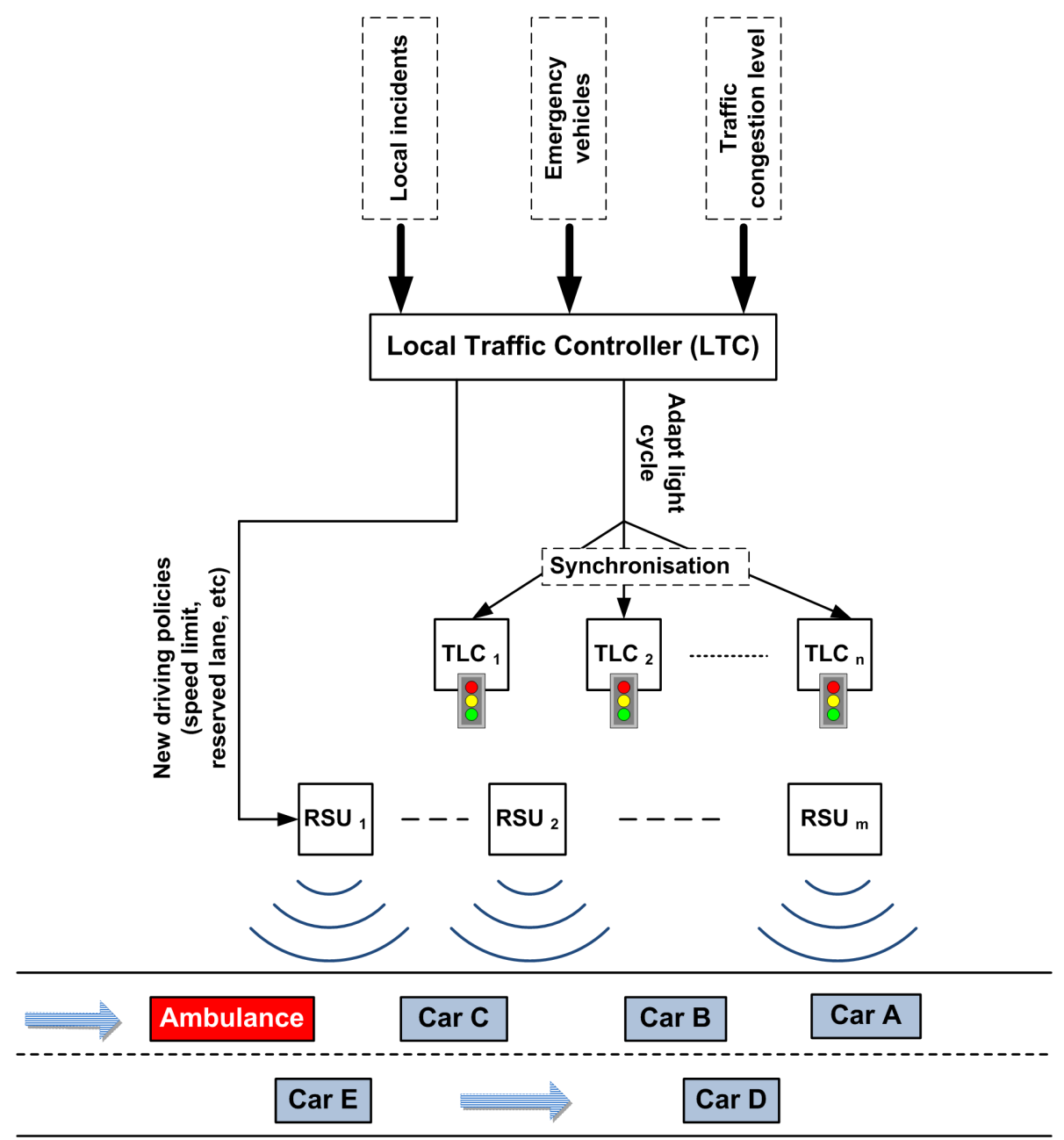

Figure 9: Example of Adaptive TMS

malicious misuse of the monitoring equipment such as wireless sensors, M2M devices and surveillance cameras. The second category consists of the attacks launched against the smart vehicles being used as source/destination of traffic information by spreading forged information about traffic congestion level, incidents etc. Finally, the last category includes the attacks aiming at breaking down the key components needed for TMS operations (i.e. the core system which manages both the road infrastructure and the monitoring equipment). Cyber attacks are the most severe threats for the core system of TMS since a successful infiltration, through any cyber defence breaches, will give criminals full control of the transportation infrastructure, which would cause massive loss of data and serious damage to physical assets in addition to potential human lives loss. Several worms have been developed to launch cyber attacks against critical systems such as the "Stuxnet" worm, "Duqu", "Flame" and "Gauss" viruses. To cope with the increasing threat of these sophisticated worms, conventional security solutions such as anti-virus softwares and firewalls are, unfortunately, not sufficient. Therefore, more robust countermeasures are needed to defeat these cyber attacks.

Besides the above security threats, the recent trends of using smart phones as traffic probes for more accurate traffic congestion estimation have raised a particular concern about the privacy of the users. In the context of a TMS, the use of smartphones entails also the risk that anyone can join the system and start sending its location samples. This means that the system is exposed to potential reporting of forged location data by misbehaving users, which may lead to inaccurate assessment of the real traffic conditions. Consequently, erroneous traffic information will be spread by the TMS towards the drivers resulting in traffic conditions deterioration as well as traffic incidents in some extreme cases. Mobile users location privacy and the threats against it have recently raised an increasing attention due to the numerous locationaware applications that have been designed for smartphones. To protect their privacy, the drivers tend usually to have their exact position obfuscated to prevent being tracked by a third party. This obfuscation will significantly reduce the accuracy of real-time traffic conditions estimation. Therefore, robust privacy preservation techniques are required to reassure the users and incite them to disclose their exact position.

Trust management of mobile users is another issue for TMS in order to deliver reliable decisions and ensure better control of the traffic flow. The trust management task will 
enable the TMS to establish a list of reliable mobile data sources, according to periodic evaluation of their trust level, in addition to roads monitoring equipment such as, sensors, CCTV cameras, induction loops etc. A misbehaving driver (e.g. terrorist, robber) may use his smartphone to broadcast fake information in order to re-route the other cars to clear the way for the terrorists'/robbers' vehicle, or just divert the traffic towards a specific road segment to create a bottleneck. As the TMS will not react to information sent by a non-trusted data source, the misbehaving driver may spoof the identity of another reliable data source to ensure that his goal will be achieved. In this case, the lack of adequate authentication mechanisms will be of detrimental impact.

\section{RELATED EUROPEAN/INTERNATIONAL INITIATIVES} (PROJECTS)

In this section, we present a snapshot of recent projects that aim to improve the different aspects of a traffic management system. The projects have been organised based on their major concern in terms of architecture, safety, efficiency, sustainability and energy-awareness, reliability and security and innovative services. Table IV summarises these projects.

\section{A. Architecture}

The Keystone Architecture Required for European Networks (KAREN) project [110] made the first steps towards an integrated ITS architecture between 1998 and 2000. KAREN has addressed the need for a single reference platform in Europe, which would provide a basis for the development of ITS products and services. The Framework Architecture Made for Europe (FRAME-NET) project [111] has gathered a thematic network of interested parties funded by the European Union Fifth Framework programme (FP5), which have coordinated and promoted wide scale implementation of ITS architecturerelated activities in Europe starting from July 2001. The Framework Architecture Made for Europe - Support (FRAME$S$ ) [111] has extended the original ITS architecture and updated it to include the latest requirements from functional, physical and communications points of view.

Extending the FRAME architecture (E-FRAME) [111] is another three year European-funded project which has further extended the FRAME ITS architecture to support the creation of inter-operable and scalable cooperative systems throughout the European Union. The project which started in 2008 has focused on acquiring, exchanging, and processing data from vehicles (e.g. road conditions) for the benefit of the driver (e.g. better driver information and trip planning) and third parties (e.g. knowledge of road network state). Very important is the integration role of this project as the updated ITS architecture includes cooperative systems services and applications developed by other European projects such as COOPERS [113], CVIS [121] and SAFESPOT [114]. Also the Preparation for Driving Implementation and Evaluation of C2X Communication Technology (PREDRIVE C2X) FP7 integrated project [64] has established a pan-European architecture framework for cooperative systems, setting the road for field operational tests on cooperative systems by focusing on architectural design, implementation and deployment aspects. The very recent project Accelerate Cooperative Mobility (DRIVE C2X) [64] goes beyond the previous projects which have proven the feasibility of safety and traffic efficiency applications based on vehicular communications and addresses large-scale field trials under real-world conditions at multiple national test sites across Europe.

\section{B. Safety}

The COOPerative SystEMS for Intelligent Road Safety (COOPERS) [113] is a research and development project in the area of cooperative and in-vehicle integrated safety systems funded by the European Commission FP6 programme in 2006. COOPERS focuses on the development of innovative telematics applications based on communication between vehicles and infrastructure, which will bring together experts from both car industry and infrastructure operators. Ultimately the goal of COOPERS is the enhancement of road safety by direct and up to date traffic information communication between infrastructure and motorised vehicles.

The Cooperative Vehicles and Road Infrastructure for Road Safety (SAFESPOT) [114] is an FP6 integrated research project co-funded by the European Commission Information Society Technologies programme. SAFESPOT focuses on road accidents prevention via an online assistant which extends the drivers' awareness of the surroundings in both space and time and detects potentially dangerous situations in advance. SAFESPOT makes use of vehicle to vehicle and vehicle to infrastructure communications. The European Commissionfunded Network of Excellence on Advanced Passive Safety (APSN) [128] has established an integrated European Virtual Centre of Excellence on vehicle passive safety research and development in 2006. APSN goal was to accelerate the improvements in road safety in order to reduce the annual road victims in the European Union. Advanced Protection SYStems (APROSYS) [129] is a FP6 integrated project that has developed and introduced critical scientific and technology developments that improve passive safety for road users in all-relevant accident types in Europe. The Save Our Lives - A Comprehensive Road Safety Strategy for Central Europe (SOL) [112] is an on-going Central European project whose goal is to promote sustainable mobility, increase awareness for safety issues, and contribute to the achievement of higher quality of living conditions for road users.

\section{Sustainability and Energy-awareness}

The "Partners for Advanced Transportation TecHnology (PATH)" [68] multi-disciplinary large scale research and development program, which involves collaboration between universities, private industry, state and local agencies, and non-profit institutions from California, USA. PATH proposes state of the art research solutions to the surface transportation systems problems. PATH focuses on the relatively longterm, high-impact solutions, and on the evolutionary steps that are required to have the long-term solutions deployed. Some of PATH research focuses on fuel saving and transportrelated gas emissions reduction. The Connect and Drive 
[115] is a collaborative project between Dutch companies and universities, sponsored by the Dutch Government, which has developed technologies for Cooperative Advanced Cruise Control (CACC). CACC has extended the functionality of the Adaptive Cruise Control (ACC) based on communication between vehicles in addition to sensor capabilities of each vehicle to adapt the speed to other vehicles. The goal of the project was to optimize traffic throughput, improve traffic safety and reduce emission of vehicles.

The Cooperative Mobility Systems and Services for Energy Efficiency (eCoMove) [119] is an FP7 European Commissionfunded project which makes use of the latest vehicle-toinfrastructure and vehicle-to-vehicle communication technologies in order to create an integrated energy-saving road traffic solution. eCoMove includes eco-driving support and ecotraffic management in its endeavour to reduce energy waste by passenger and goods vehicles. Lately, there is a significant push towards Full Electric Vehicles (FEV) and many FEVrelated research projects are on-going. Among these works the Combining Infrastructure for Efficient Electric Mobility (eCo$F E V$ ) [120] is a FP7 European Commission funded project which aims at achieving a breakthrough in the FEV space by proposing a general architecture for integration of FEV into the different cooperating infrastructure systems. This architecture makes use of state of the art communications technologies in order to support precise FEV telematics and charging management services based on the real-time information. The project will complete in 2015.

\section{Efficiency, Reliability and Security}

The Cooperative Vehicle Infrastructure Systems (CVIS) [121] is a large European Commission-funded FP6 integrated project that has designed, developed and tested technologies which support vehicles to communicate with each other and with the nearby road infrastructure efficiently. By using CVIS technology the vehicles can communicate the latest traffic information and safety warnings to road operators and other nearby vehicles, connecting through a multi-channel terminal with a wide range of potential carriers, including cellular networks (GPRS, UMTS), wireless local area networks (WiMax, Wi-Fi), short-range microwave beacons (DSRC) or infrared (IR) based on the international ISO CALM standards.

The Highly Dependable IP-based Networks and Services (HIDENETS) [123] is a FP6 European Commission-funded project which has developed and analyzed end-to-end resilience solutions for distributed applications and mobilitybased services in vehicular environments. The Secure Vehicular Communications (SeVeCom) [124] is an EU-funded project that has focused on providing full definition and implementation of security requirements for vehicular communications. The project goal was to develop technologies to improve road safety and optimise road traffic by making use of vehicular to infrastructure and inter-vehicular communications. The Geoaddressing and geo-routing for vehicular communications (GeoNET) [122] is a recent European FP7 project which has focused on a geographic addressing and routing protocol with support for IPv6 to be used to deliver safety messages between vehicles and between vehicles and roadside infrastructure. This enables transparent IP connectivity between a vehicle and the infrastructure, even using multi-hop or cache-based solutions.

\section{E. Innovative Services}

The Adaptive Integrated Driver vEhicle interface (AIDE) [125] is an European project which has designed, developed and validated an adaptive driver-vehicle interface system that brings in the potential benefits of many new in-vehicle technologies and nomad devices in terms of mobility and comfort in an efficient and integrated manner, without compromising safety. The Integrated Wireless and Traffic Simulation Platform for Real-Time Road Traffic Management Solutions (iTETRIS) [65] is an European FP7-funded project which has developed an open, ETSI standard compliant, and flexible simulation platform that integrates wireless communications and road traffic simulation technologies and solutions in a common environment that is easily tailored to specific situations allowing performance analysis of cooperative ITS at the level of a city.

The Developing Next Generation Intelligent Vehicular Networks and Applications (DIVA) is an on-going Canadian NSERC-funded research network which targets the development and integration of communication systems, vehicular technologies, and applications for enabling nationwide deployment of vehicular ad-hoc networks and intelligent transportation systems. Its focus ranges from developing innovative large-scale communication architectures and wireless network technologies to proposing solutions increasing the efficiency and safety of Canada's transportation systems.

The Road Safety Attributes Exchange Infrastructure in Europe (ROSATTE) is a FP7 European Commission-funded project which has defined and implemented infrastructure and supporting tools to ensure the efficiency and quality assurance in the data supply chain from public authorities to commercial map providers with regards to safety related road content. The on-going IBM Smarter City [69] project includes a large variety of initiatives, including traffic management, on a global scale. In particular traffic-related research and development focuses on traffic modelling and simulation, smarter parking, maximizing revenue and minimizing environmental impact, integrated fare management, real-time traffic updates, reducing the commute time, improving mobility within a city, etc. The overall aim is to contribute towards realising a smarter transportation system for the 21 st century.

\section{Open Challenges}

This section identifies some open challenges existing research in the area of TMS for smart cities faces and discusses potential avenues to putting additional effort toward finding highly sought after solutions. These challenges are presented in terms of the following major stages related to traffic data: gathering, storage, aggregation, exchange, processing and application-layer support.

A significant challenge in terms of information gathering is related to the number of entities which collect trafficbased data, from road traffic operators such as public transport companies, private taxi companies, etc., and public traffic 
Table IV: Major ITS-related Projects

\begin{tabular}{|c|c|c|c|}
\hline Project & Time period & Area of concern & Reference \\
\hline KAREN (Keystone Architecture Required for European Networks) & $1998-2000$ & \multirow[t]{7}{*}{ Architecture } & {$[110]$} \\
\hline FRAME-NET (Framework Architecture made for Europe) & 2008-2011 & & [111] \\
\hline CarCoDe (Platform for Smart Car to Car Content Delivery) & $2012-2015$ & & {$[66]$} \\
\hline COOPERS (Cooperative systems for Intelligent Road Safety) & 2006-2010 & & [113] \\
\hline CVIS (Cooperative Vehicle Infrastructure Systems) & $2006-2010$ & & {$[121]$} \\
\hline SAFESPOT (Cooperative Vehicles and Road Infrastructure) & 2006-2010 & & {$[114]$} \\
\hline DRIVE C $2 \mathrm{X}$ & $2010-2013$ & & {$[64]$} \\
\hline APSN (Network of excellence on advanced passive safety) & 2004-2008 & \multirow[t]{3}{*}{ Safety } & {$[128]$} \\
\hline APROSYS (Advanced protection systems) & 2004-2009 & & {$[129]$} \\
\hline SOL (Save Our Lives:A comprehensive road safety strategy for central europe) & $2007-2013$ & & {$[112]$} \\
\hline PATH (Partners for advanced transportation technology) & 1986-ongoing & \multirow{4}{*}{$\begin{array}{l}\text { Sustainability and } \\
\text { Energy-awareness }\end{array}$} & {$[68]$} \\
\hline CACC (Cooperative Advanced Cruise Control) & 2009-ongoing & & {$[115]$} \\
\hline eCoMove (Cooperative Mobility Systems and Services for energy efficiency) & $2010-2013$ & & [119] \\
\hline eCo-FEV (Combining infrastructure for efficient electric mobility) & $2012-2015$ & & {$[120]$} \\
\hline HIDENETS (Highly dependable IP-based networks and services) & 2006-2009 & \multirow{3}{*}{$\begin{array}{l}\text { Efficiency, Reliability } \\
\text { and Security }\end{array}$} & {$[123]$} \\
\hline SeVeCom (Secure Vehicular Communications) & 2006-2009 & & {$[124]$} \\
\hline GeoNET (The geo-addressing and geo-routing for vehicular communications) & $2008-2010$ & & {$[122]$} \\
\hline AIDE (Adaptive integrated driver vehicle interface) & 2004-2008 & \multirow[t]{7}{*}{ Innovative Services } & {$[125]$} \\
\hline $\begin{array}{l}\text { Itetris (The integrated wireless and traffic simulation platform for real-time and road } \\
\text { traffic management solutions) }\end{array}$ & $2008-2011$ & & {$[65]$} \\
\hline DIVA (The developing next generation intelligent vehicular networks adn applications) & 2012- ongoing & & [62] \\
\hline ROSATTE (Road Safety Attributes Exchange Infrastructure in Europe) & $2008-2010$ & & {$[126]$} \\
\hline IBM Smarter Cities & & & {$[69]$} \\
\hline FOTsis (Field Operational test on safe intelligent and sustainable road operation) & $2010-2013$ & & {$[63]$} \\
\hline CopITS (Cooperative cars and roads for safer and intelligent transport systems) & 2010-2013 & & [67] \\
\hline
\end{tabular}

management authorities such as local councils, planning institutions, etc., to health and environment monitoring institutions, such as health boards, environmental protection agencies, etc. and private companies and individuals. All these data gathering entities use independent measuring methods which acquire various data with different characteristics and using diverse methodologies and save it in their own databases. Relative simple issues such as data formatting in the absence of a general accepted standard for the representation of trafficrelated data results in significant problems for its potential utilization by third parties. The most important consequence of this lack of a common format is the difficult synchronization of the information gathered by various sources, which makes almost impossible coherent usage of information and crosscorrelation of events. There are steps forward in this direction by proposing standards for representation and storage, but their adoption is very limited to date.

Data storage suffers from the same problems and has the same open challenges with information gathering, as these two stages are highly inter-connected. The only issue which is strictly related to storage is the database support. In this regard there are several widely researched solutions, of which the XML-based ones are the most popular. Open challenges are mostly performance-related, especially in a distributed environment.

Data aggregation poses additional challenges to those related to data gathering and storage. As the data originates from different sources, their conversion is the most important next step. In this process, the first obstacle is the amount of data collected which is increasing exponentially and the second its 
complexity. This makes data conversion increasingly difficult and highly time and resource consuming. In this stage relevant data extraction and cleaning, as well as data reduction might be required. Each of these tasks has its own challenges including defining what is relevant and what is noise, identifying one or the other and extracting the useful data, given certain accuracy expectations. The latest interest surge in big data research provides solutions to be also used in TMS for smart cities.

Data exchange has attracted significant attention from many interested parties and has resulted in the proposal, design and standardisation of communication protocols of which IEEE 1609 DSRC/WAVE [127] and IEEE 802.11p [40] are best known. Yet, there are still open challenges. Some challenges are related to routing, difficult to address due to both high speed of vehicles and their increased mobility which make existing solutions difficult to be applied. Other problems concern security, and are related to driver or passenger authentication, confidentiality and availability. Not at last supporting high levels of Quality of Service for data exchange is highly difficult to achieve, mostly as data transfer in multi-hop, high mobility node environments severely affects throughput, delay and jitter.

Data processing and application-layer service support have many open challenges, mostly application specific. With the increase in the number and type of such applications, the list of open challenges also grows, with issues mostly related to the real-time processing of large amounts of data, real-time interaction with users and group of users, user profiling and modeling, interaction with the environment, etc.

\section{CONCLUSION}

Improving the efficiency of Traffic Management Systems (TMS) is still an active and challenging research area due to the criticality of transportation infrastructure being monitored by such systems. This survey has provided a comprehensive study of the different phases of a modern TMS, emphasizing the main challenges and shortcomings of the existing systems and suggesting some directions to make the TMSs more efficient in future smart cities. First, we have presented the different existing technologies used for traffic data gathering and highlighted the main new technologies that can significantly improve the accuracy of the collected data. We have also surveyed the numerous routing protocols used in VANETs to disseminate the collected data among vehicles and shown their respective advantages and shortcomings. The congestion problem in VANETs as well as the simulation tools are also deeply discussed. Second, a critical discussion of data fusion and aggregation solutions are provided along with a brief overview on the TMDD standard used by IBM. Third, routes planning and traffic prediction services are investigated with main focus on highlighting the limitations of the existing algorithms and suggesting alternative directions for better accuracy and efficiency. Finally, we presented our vision on improving TMSs efficiency and robustness, which consists in leveraging smart vehicles capabilities and advanced parking systems to achieve the desired level of accuracy and control of the traffic. Moreover, the security threats targeting TMSs, the open challenges need to be addressed as well as the major international research projects dealing with TMS related challenges are presented.

\section{ACKNOWLEDGEMENT}

This work was supported, in part, by Science Foundation Ireland grant 10/CE/I1855 to Lero - the Irish Software Engineering Research Centre (www.lero.ie).

\section{REFERENCES}

[1] European Initiative on Smart Cities, 2010-2020, http://setis.ec.europa.eu/set-plan-implementation/technologyroadmaps/european-initiative-smart-cities

[2] D. B. Johnson and D. A. Maltz, 'The Dynamic Source Routing Protocol for Mobile Ad Hoc Networks', (Internet-Draft), Mobile Ad-hoc Network (MANET) Working Group, IETF, October 1999.

[3] F. Li and Y. Wang, "Routing in vehicular ad hoc networks: A survey," Vehicular Technology Magazine, IEEE, vol. 2, no. 2, pp. 12-22, 2007.

[4] R. A. Santos, A. Edwards, and O. Alvarez, "Towards an Inter-vehicle Communication Algorithm," in Electrical and Electronics Engineering, 2006 3rd International Conference on, 2006, Veracruz, Mexico, pp. 1-4.

[5] O. K. Tonguz, N. Wisitpongphan, and F. Bai, "DV-CAST: A distributed vehicular broadcast protocol for vehicular ad hoc networks," Wireless Communications, IEEE, vol. 17, no. 2, pp. 47-57, 2010.

[6] Y. Ding, C. Wang, and L. Xiao, "A static-node assisted adaptive routing protocol in vehicular networks," in Proceedings of the fourth ACM international workshop on Vehicular ad hoc networks, 2007, Montreal, Canada, pp. 59-68.

[7] J. Zhao and G. Cao, "VADD: Vehicle-Assisted Data Delivery in Vehicular," Vehicular Technology, IEEE Transactions on, vol. 57, no. 3, pp. 1910-1922, 2008.

[8] G. Korkmaz, E. Ekici, F. zgner, and . zgner, "Urban multi-hop broadcast protocol for inter-vehicle communication systems," in Proceedings of the 1st ACM international workshop on Vehicular ad hoc networks, 2004, Philadelphia, PA, USA, pp. 76-85.

[9] E. Fasolo, A. Zanella, and M. Zorzi, "An effective broadcast scheme for alert message propagation in vehicular ad hoc networks," in Communications, 2006. ICC06. IEEE International Conference on, 2006, vol. 9, Istanbul, Turkey, pp. 3960-3965.

[10] G. Korkmaz, E. Ekici, and F. Ozguner, "An efficient fully ad-hoc multihop broadcast protocol for inter-vehicular communication systems," in Communications, 2006. ICC06. IEEE International Conference on, 2006, vol. 1, Istanbul, Turkey, pp. 423-428.

[11] S. A. Arzil, M. H. Aghdam, and M. A. J. Jamali, "Adaptive routing protocol for VANETs in city environments using real-time traffic information," in Information Networking and Automation (ICINA), 2010 International Conference on, 2010, vol. 2, Kunming, China, pp. V2-132.

[12] V. Naumov and T. R. Gross, "Connectivity-aware routing (CAR) in vehicular ad-hoc networks," in INFOCOM 2007. 26th IEEE International Conference on Computer Communications. IEEE, 2007, Anchorage , Alaska , USA, pp. 1919-1927.

[13] M. Durresi, A. Durresi, and L. Barolli, "Emergency broadcast protocol for inter-vehicle communications," in Parallel and Distributed Systems, 2005. Proceedings. 11th International Conference on, 2005, vol. 2, Fuduoka, Japan, pp. 402-406.

[14] J. Gong, C. Z. Xu and H. J, "Predictive Directional Greedy Routing in Vehicular Ad hoc Networks," In Proc. of the International Conference on Distributed Computing Systems Workshops (ICDCSW'07), June 25-29, 2007, Toronto, Canada. pp 2-2.

[15] U. Nagaraj and P. Dhamal, "Broadcasting Routing Protocols in VANET," Network and Complex Systems, vol. 1, no. 2, pp. 13-19, 2012.

[16] M. T. Sun, W. C. Feng, T. H. Lai, K. Yamada, H. Okada, and K. Fujimura, "GPS-based message broadcasting for inter-vehicle communication," in Parallel Processing, 2000. Proceedings. 2000 International Conference on, 2000, Toronto, Canada, pp. 279-286.

[17] B. Karp and H. T. kung, "GPSR: Greedy perimeter Stateless Routing for Wireless Networks", ACM MobiCom 2000, August 6-11, 2000, Boston, MA, USA, pp. 243-254.

[18] Z. Mo, H. Zhu, K. Makki, and N. Pissinou, "MURU: A multi-hop routing protocol for urban vehicular ad hoc networks," in Mobile and Ubiquitous Systems: Networking \& Services, 2006 Third Annual International Conference on, 2006, London, Great Britain, pp. 1-8. 
[19] B. C. Seet, G. Liu, B. S. Lee, C. H. Foh, K. J. Wong, and K. K. Lee, "A-STAR: A mobile ad hoc routing strategy for metropolis vehicular communications," NETWORKING 2004. Networking Technologies, Services, and Protocols; Performance of Computer and Communication Networks; Mobile and Wireless Communications, pp. 989-999, 2004.

[20] J. Bernsen and D. Manivannan, "Unicast routing protocols for vehicular ad hoc networks: A critical comparison and classification," Pervasive and Mobile Computing, vol. 5, no. 1, pp. 1-18, 2009.

[21] A. Bachir and A. Benslimane, "A multicast protocol in ad hoc networks inter-vehicle geocast," in Vehicular Technology Conference, 2003. VTC 2003-Spring. The 57th IEEE Semiannual, 2003, vol. 4, Jeju, Korea, pp. 2456-2460.

[22] M. Jerbi, R. Meraihi, S. M. Senouci, and Y. Ghamri-Doudane, "GyTAR: improved greedy traffic aware routing protocol for vehicular ad hoc networks in city environments," in Proceedings of the 3rd international workshop on Vehicular ad hoc networks, 2006, Los Angeles, CA, USA pp. $88-89$

[23] W. Sun, H. Yamaguchi, K. Yukimasa, and S. Kusumoto, "GVGrid: A QoS routing protocol for vehicular ad hoc networks," in Quality of Service, 2006. IWQoS 2006. 14th IEEE International Workshop on, 2006, New Haven, CT, USA, pp. 130-139.

[24] H. Lu and C. Poellabauer, "Balancing broadcast reliability and transmission range in VANETs," in Vehicular Networking Conference (VNC), 2010 IEEE, 2010, Jersey City, New Jersey, USA, pp. 247-254.

[25] M. Feiri, J. Petit, and F. Kargl, "Congestion-based certificate omission in VANETs," in Proceedings of the ninth ACM international workshop on Vehicular inter-networking, systems, and applications, 2012, Ambleside, United Kingdom, pp. 135-138.

[26] D. B. Rawat, D. C. Popescu, G. Yan, and S. Olariu, "Enhancing VANET Performance by Joint Adaptation of Transmission Power and Contention Window Size," IEEE Transactions on Parallel and Distributed Systems, vol. 22, no. 9, pp. 1528-1535, Sep. 2011.

[27] S. Djahel and Y. Ghamri-Doudane, "A Robust Congestion Control Scheme for Fast and Reliable Dissemination of Safety Messages in VANETs", IEEE WCNC 2012, Paris, France, pp. 2264-2269, April 1-4, 2012.

[28] S. Djahel, M. Salehie, I. Tal and P. Jamshidi, " Adaptive Traffic Management for Secure and Efficient Emergency Services in Smart Cities", IEEE Pervasive Computing and Communication (PerCom) conference, San Diego, California, USA, pp. 240-243, March 18-22, 2013.

[29] I. H. Brahmi, S. Djahel and Y. Ghamri-Doudane. "A Hidden Markov Model based Scheme for Efficient and Fast Dissemination of safety Messages in VANETs". IEEE GLOBECOM 2012, Anaheim, California, USA, pp. 177-182, December 3-7, 2012.

[30] V. T. Ngoc Nha, S. Djahel and J. Murphy. "A Comparative Study of Vehicles' Routing Algorithms for Route Planning in Smart Cities". VTM 2012, Satellite Workshop of IFIP Wireless Days 2012, Dublin, Ireland, pp. 1-6, November 20, 2012.

[31] H. I. Brahmi, S. Djahel and J. Murphy. "Improving Emergency Messages Transmission Delay in Road Monitoring based WSNs". The 6th Joint IFIP Wireless and Mobile Networking Conference (WMNC'2013), Dubai, UAE, pp. 1-8, April 23-25, 2013.

[32] N. D. Lane, E. Miluzzo, H. Lu, D. Peebles, T. Choudhury and A. T. Campbell. "A Survey of Mobile Phone Sensing". IEEE Communications Magazine, Vol. 48, N. 9, pp.140-150, September 2010.

[33] R. K. Ganti, F. He and H. Lei. "Mobile crowdsensing: current state and future challenges". IEEE Communications Magazine, Vol. 49, N. 11, pp.32-39, November 2011.

[34] S. konur and M. Fisher, "Formal Analaysis of a VANET Congestion Control Protocol through Probabilistic Verification", In Proc. of the $73^{r d}$ IEEE Vehicular Technology Conference (VTC Spring), Budapest, Hungary, pp. 1-5, May 15-18, 2011.

[35] M. Torrent-Moreno, Jens Mittag, P. Santi and H. Hartenstein, "Vehicleto-Vehicle Communication: Fair Transmit Power Control for SafetyCritical Information", IEEE Transactions on Vehicular Technologty, Vol. 58, No. 7, pp. 3684-3703, Sep. 2009.

[36] M. Bouassida and M. Shawky, "A Cooperative and Fully-distributed Congestion Control Approach within VANETs", In Proc. of the $9^{\text {th }}$ International Conference on Intelligent Transport systems Telecommunications, (ITST), Lille, France, pp. 526-531, 20-22 Oct. 2009.

[37] M. D. Felice, K. R. Chowdhury and L. Bononi, "Analyzing the Potential of Cooperative Cognitive Radio Technology on Inter-Vehicle Communication", In Proc. of IFIP Wireless Days 2010, Venice, pp. 1-6, Oct. 20-22, 2010.

[38] K. Fawaz, A. Ghandour, M. Olleik and H.Artail,'Improving reliability of safety applications in vehicle ad hoc networks through the implementation of a cognitive network ", In Proc. of the $17^{\text {th }}$ International
Conference on Telecommunications (ICT) , Doha, Qatar, pp. 798-805, Apr. 4-7, 2010

[39] X. Y. Wang and P. Han Ho, ”A Novel sensing Coordination Framework for CR-VANET", IEEE Transactions on Vehicular Technology, Vol. 59, No. 4, pp. 1936-1948, May. 2010.

[40] 802.11p-2010 - IEEE Standard for Information technology- Local and metropolitan area networks- Specific requirements- Part 11: Wireless LAN Medium Access Control (MAC) and Physical Layer (PHY) Specifications Amendment 6: Wireless Access in Vehicular Environments.

[41] R. Aquino-Santos, et al., "Inter-Vehicular Communications Using Wireless Ad Hoc Networks", Automotive Informatics and Communicative Systems: Principles in Vehicular Networks and Data Exchange. IGI Global, 2009. pp. 120-138. doi:10.4018/978-1-60566-338-8.ch007

[42] L. Husheng and D. K. Irick, "Collaborative Spectrum Sensing in Cognitive Radio Vehicular Ad Hoc Networks: Belief Propagation on Highway", In Proc. of the $71^{\text {st }}$ IEEE Vehicular Technology Conference, VTC Spring 2010, Taipei, pp. 1-5, May. 16-19, 2010.

[43] C.L.P. Chen, J. Zhou and W. Zhao, "A Real-Time Vehicle Navigation Algorithm in Sensor Network Environments," IEEE Transactions on Intelligent Transportation Systems, Vol.13, No.4, pp.1657,1666, December 2012.

[44] T.-Y. Liao and T.-Y. Hu, An object-oriented evaluation framework for dynamic vehicle routing problems under real-time information, Expert Systems with Applications, vol. 38, pp. 12548-12558, Sept. 2011.

[45] M. Gendreau, J.-Y. Potvin, O. Braumlaysy, G. Hasle, and A. L kketangen, Metaheuristics for the Vehicle Routing Problem and Its Extensions: A Categorized Bibliography, in The Vehicle Routing Problem: Latest Advances and New Challenges (B. Golden, S. Raghavan, and E. Wasil, eds.), vol. 43 of Operations Research/Computer Science Interfaces, pp. 143-169, Boston, MA: Springer US, 2008.

[46] B. Tatomir, L. J. M. Rothkrantz, and A. C. Suson, Travel time prediction for dynamic routing using ant based control, in Winter Simulation Conference, Austin, TX, USA, pp. 1069-1078, Dec. 2009.

[47] H. Kanoh and K. Hara, Hybrid genetic algorithm for dynamic multiobjective route planning with predicted trafic in a real-world road network, in Proceedings of the 10th annual conference on Genetic and evolutionary computation - GECCO 08, (New York, NY, USA), p. 657, ACM Press, July 2008.

[48] B. Chakraborty, T. Maeda, and G. Chakraborty, Multiobjective route selection for car navigation system using genetic algorithm, Proceedings of the 2005 IEEE Midnight-Summer Workshop on Soft Computing in Industrial Applications, 2005. SMCia/05., Espoo, Finland, pp. 190195, 2005.

[49] S. Mathur, T. Jin, N. Kasturirangan, J. Chandrasekaran, W. Xue, M. Gruteser, and W. Trappe, "Parknet: drive-by sensing of road-side parking statistics," in Proceedings of the 8th international conference on Mobile systems, applications, and services, 2010, San Francisco, CA, USA, pp. 123-136.

[50] A. Klappenecker, H. Lee, and J. L. Welch, "Finding available parking spaces made easy," Proceedings of the 6th International Workshop on Foundations of Mobile Computing, 2012, Cambridge, MA, USA,pp. 4952.

[51] R. Panayappan, "VANET-based Approach for Parking Space Availability.", in VANET '07 Proceedings of the fourth ACM international workshop on Vehicular ad hoc networks, 2007, Montreal, Canada, pp. $75-76$

[52] E. Kokolaki, M. Karaliopoulos, and I. Stavrakakis, "Opportunistically assisted parking service discovery: Now it helps, now it does not," Pervasive and Mobile Computing, vol. 8, no. 2, pp. 210-227, 2012.

[53] T. Delot, N. Cenerario, S. Ilarri, and S. Lecomte, "A cooperative reservation protocol for parking spaces in vehicular ad hoc networks," in Proceedings of the 6th International Conference on Mobile Technology, Application \& Systems, 2009, Nice, France, p. 30.

[54] M. Caliskan, A. Barthels, B. Scheuermann, and M. Mauve, "Predicting parking lot occupancy in vehicular ad hoc networks," in Vehicular Technology Conference, 2007. VTC2007-Spring. IEEE 65th, 2007 Dublin, Ireland, pp. 277-281.

[55] M. Caliskan, D. Graupner, and M. Mauve, "Decentralized discovery of free parking places," in Proceedings of the 3rd international workshop on Vehicular ad hoc networks, New York, NY, USA, 2006, pp. 30-39.

[56] P. Szczurek, B. Xu, O. Wolfson, J. Lin, and N. Rishe, "Learning the relevance of parking information in VANETs," in Proceedings of the seventh ACM international workshop on VehiculAr InterNETworking, 2010, Chicago, IL, USA, pp. 81-82.

[57] S. Evenepoel, J. Van Ooteghem, S. Verbrugge, D. Colle and M. Pickavet, "On-street smart parking networks at a fraction of their cost: 
performance analysis of a sampling approach," ETT Special Issue on Smart Cities, vol. 25, no. 1, pp. 136-149, Jan. 2014.

[58] W. Min and L. Wynter, "Real-time road traffic prediction with spatiotemporal correlations", Transportation Research Part C: Emerging Technologies 19(4), pp. 606-616, Elsevier, 2011

[59] J. He et al., 'Ensemble-based Method for Task 2: Predicting Traffic Jam", in Proc. of IEEE International Conference on Data Mining Workshops, Sydney, Australia, 14-17 December 2010, pp. 1363-1365.

[60] Y. Kamarianakis, W. Shen and L. Wynter, " Real-time road traffic forecasting using regime-switching space-time models and adaptive LASSO", Applied Stochastic Models in Business and Industry, Wiley Online Library, 2012.

[61] W. Shen and L. Wynter, "Real-time road traffic fusion and prediction with GPS and fixed-sensor data", Information Fusion (FUSION), 15th International Conference on, Singapore, pp. 1468-1475, 2012.

[62] O. Abumansoor, and A. Boukerche. "Preventing a dos threat in vehicular ad-hoc networks using adaptive group beaconing." Proceedings of the $8 \mathrm{~h}$ ACM symposium on QoS and security for wireless and mobile networks. ACM, 2012, Paphos, Cyprus, pp. 63-70.

[63] Viragg, L., Koves, J., \& Edelmayer, A. (2013, March). Extension of the ITS Station Architecture to Low-Power Pervasive Sensor Networks. In Advanced Information Networking and Applications Workshops (WAINA), 2013 27th International Conference on (pp. 1386-1391). IEEE. Barcelona, Spain.

[64] R. Stahlmann, et al. "Starting European field tests for Car-2-X communication: the DRIVE C2X framework." Proceedings 18th ITS World Congress and Exhibition. 2011, Orlando, FL, USA.

[65] V. Kumar, L. Lin, D. Krajzewicz, F. Hrizi, O. Martinez, J. Gozalvez, \& R. Bauza. (2010, May). itetris: Adaptation of its technologies for large scale integrated simulation. In Vehicular Technology Conference (VTC 2010-Spring), 2010 IEEE 71st, Taipei, Taiwan, (pp. 1-5). IEEE.

[66] CarCoDe website, "Platform for Smart Car to Car Content Delivery", http://www.itea2.org/project/index/view/?project=10147

[67] F. Filali , H. Menouar, and A. Abu-Dayya. "CopITS: The first connected car standard-compliant platform in Qatar and the region." Qatar Foundation Annual Research Forum. No. 2012.

[68] Shladover, Steven E. "PATH at 20-History and major milestones." IEEE Transactions on intelligent transportation systems 8.4 (2007): 584-592.

[69] Naphade, M., Banavar, G., Harrison, C., Paraszczak, J., \& Morris, R. (2011). Smarter cities and their innovation challenges. Computer, 44(6), 32-39.

[70] E. M. Daly, F. Lecue and V. Bicer, "Westland Row Why So Slow? Fusing Social Media and Linked Data Sources for Understanding RealTime Traffic Conditions", In Proc. of the 2013 international conference on Intelligent user interfaces, IUI'13, March 19-22, 2013, Santa Monica, CA, USA. pp. 203-212.

[71] F. Lecue, A. Schumann, and M. L. Sbodio," Applying semantic web technologies for diagnosing road traffic congestions", In Proc. of the 11 th International Semantic Web Conference (ISWC) (2012), November 11-15, Boston, USA, pp. 114-130.

[72] M. Tubaishat, P. Zhuang, Q. Qi, and Y. Shang, "Wireless Sensor Networks in Intelligent Transportation Systems", Wireless Communications and Mobile Computing, March 2009.

[73] M. Tubaishat, Q. Qi, Y. Shang and H. Shi. "Wireless Sensor-Based Traffic Light Control", IEEE Consumer Communications and Networking Conference (CCNC'08), Jan 2008, Las Vegas, NV, pp. 702-706.

[74] M. Tubaishat, Y. Shang and H. Shi,'Adaptive Traffic Light Control with Wireless Sensor Networks"1, IEEE Consumer Communications and Networking Conference (CCNC'07), Jan 2007, Las Vegas, NV, pp. $187-$ 191.

[75] A. Bachir, M. Dohler, T. Watteyne, and K. K. Leung, "MAC Essentials for Wireless Sensor Networks", IEEE Communications Surveys \& Tutorials, vol. 12, no. 2, pp. 222-2481, 2010.

[76] I. F. Akyildiz, W. Su, Y. Sankarasubramaniam, and E. Cayirci, "Wireless sensor networks: a survey", Computer Networks, vol. 38, no. 4, pp. 393422, 2002.

[77] J. Yick, B. Mukherjee, and D. Ghosal, "Wireless sensor network survey", Computer Networks, vol. 52, no. 12, pp. 2292-2330, 2008

[78] S. Joerer, C. Sommer and F. Dressler, "Towards Reproducibility and Comparability of IVC Simulation Studies: A Literature Survey", IEEE Communications Magazine, vol. 50, no. 10, pp. 82-88, 2012.

[79] R. Stanica, E. Chaput, and A. Beylot, "Properties of the MAC layer in safety vehicular Ad Hoc networks". IEEE Communications Magazine, vol. 50, no. 5, pp. 192-200, 2012.

[80] ETSI TS 102 637-2, "Intelligent Transport Systems (ITS); Vehicular Communications; Basic Set of Applications; [Part 2: Specification of
Cooperative Awareness Basic Service;]", Draft Version 1.0.4, March 2010.

[81] ETSI TS 102 637-3, "'Intelligent Transport Systems (ITS); Vehicular Communications; Basic Set of Applications; [Part 3: Specifications of Decentralized Environmental Notification Basic Service;]", Draft Version 2.1.1, April 2010.

[82] D. Rawat et al. ,'Dynamic Adaptation of Joint transmission Power and Contention Window in VANET', In Proc. of IEEE VTC Fallo9, Anchorage, AK, September 2009, pp. 1-5.

[83] L. Wischhof and H. Rohling, "Congestion Control in Vehicular Ad Hoc Networks", In Proc. of IEEE International Conference on Vehicular Electronics and Safety, Xian, Shaan"xi, China, October 14-16, 2005, pp. 58-63.

[84] Y. Mertens, M. Wellens, and P. Mahonen, "Simulation-based Performance Evaluation of Enhanced Broadcast Schemes for IEEE802.11 based Vehicular Networks", In Proc. of IEEE VTC Spring08, Singapore, May 2008, pp. 3042-3046.

[85] X. Y. Wang and P. Han Ho, "A Novel sensing Coordination Framework for CR-VANET", IEEE Transactions on Vehicular Technology, Vol. 59, No. 4, pp. 1936-1948, May. 2010.

[86] T. Watteyne, I. Aug-Blum, M. Dohler, S. Ubda, and D. Barthel, Centroid virtual coordinates - A novel near-shortest path routing paradigm, Computer Networks, vol. 53, no. 10, pp. 1697-1711, Jul. 2009.

[87] Chabini, Ismail, and Shan Lan. "Adaptations of the A* algorithm for the computation of fastest paths in deterministic discrete-time dynamic networks.” Intelligent Transportation Systems, IEEE Transactions on 3.1 (2002): 60-74.

[88] Huang, Bo, Q. Wu, and F. B. Zhan. "A shortest path algorithm with novel heuristics for dynamic transportation networks." International Journal of Geographical Information Science 21.6 (2007): 625-644.

[89] Bell, Michael GH, et al. "Time-dependent Hyperstar algorithm for robust vehicle navigation." Transportation Research Part A: Policy and Practice (2012).

[90] Behrisch, M., Bieker, L., Erdmann, J., \& Krajzewicz, D. (2011, October). Sumo-simulation of urban mobility-an overview. In SIMUL 2011, The Third International Conference on Advances in System Simulation, 2011, Barcelona, Spain, (pp. 55-60).

[91] J. Hrri, P. Cataldi et al., " Modeling and Simulating ITS Applica- tions with iTETRIS", In Proc. of the $6^{\text {th }}$ ACM workshop on Performance monitoring and measurement of heterogeneous wireless and wired networks (ACM PM2HW2N 11), Miami Beach, FL, Oct. 2011, pp. 33-40.

[92] S. Joerer, F. Dressler and C. Sommer, "Comparing apples and oranges? trends in IVC simulations", In Proc. of the $9^{\text {th }}$ ACM international workshop on Vehicular inter-networking, systems, and applications, June 25, 2012, Low Wood Bay, Lake District, UK, pp. 27-32.

[93] C. Sommer, R. German, and F. Dressler, "Bidirectionally Coupled Network and Road Traffic Simulation for Improved IVC Analysis," IEEE Transactions on Mobile Computing, vol. 10, no. 1, pp. 3-15, Jan. 2011.

[94] D. R. Choffnes and F. E. Bustamante, "An Integrated Mobility and Traffic Model for Vehicular Wireless Networks", Proc. of the 2nd ACM International Workshop on Vehicular Ad Hoc Networks (VANET), September 2005, Cologne, Germany, pp. 69-78.

[95] M. Fellendorf, P. Vortisch, "Microscopic Traffic Flow Simulator VISSIM", in Fundamentals of Traffic Simulation, J. Barcel (ed.), International Series in Operations Research \& Management Science 145 , Springer Science+Business Media, LLC 2010.

[96] Panwai, Sakda, Charnwet Haripai, and Chakrapan Tapkwa. "Development of SIDRA-TRIP integrated GPS model to evaluate fuel consumption/emission on expressway and alternative road." 17th ITS World Congress. 2010.

[97] Y. Chen, M. H. Bell and K. Bogenberger, "Reliable Pretrip Multipath Planning and Dynamic Adaptation for a Centralized Road Navigation System". IEEE Transactions on Intelligent Transportation Systems, Vol. 8, No. 1,, March, 2007, pp. 14-20.

[98] R. Doolan and G. M. Muntean, "VANET-enabled Eco-friendly Road Characteristics-aware Routing for Vehicular Traffic",IEEE Vehicular Technology Conference (VTC), Dresden, Germany, June 2013, pp. 1-5.

[99] M. Treiber, A. Kesting, and R. E. Wilson, "Reconstructing the Traffic State by Fusion of Heterogeneous Data", Computer-Aided Civil and Infrastructure Engineering, vol. 26, no. 6, pp. 408-419, Aug. 2011.

[100] H. Rehborn, M. Koller, and B. S. Kerner, "Traffic data fusion of vehicle data to detect spatiotemporal congested patterns", 19th ITS World Congress, Vienna, Austria, 22/26 Oct. 2012.

[101] N. Chen and X. Xu, "Formation-Fusion method for urban traffic flow based on evidence theory combining with fuzzy rough set", Journal of Theoretical and Applied Information Technology, vol. 49, no. 2, 2013. 
[102] E. I. Vlahogianni, J. C. Golias and M. G. Karlaftis, "Short-term Traffic Forecasting: Overview of Objectives and Methods", Transport reviews, vol. 24, no. 5, pp.533-557, 2004.

[103] S. Ishak and H. Al-Deek, "Performance of short-term time series traffic prediction model", Journal of Transportation Engineering, vol. 128, no. 6, pp. 490-498, 2002.

[104] M. S. Ahmed and A. R. Cook, "Analysis of freeway traffic time-series data by using Box-Jenkins techniques", Transportation Research Board ,pp. 1-9, 1979.

[105] S. Quan, Y. Xiuqing and G. Weikang, "A New Combination Rules of Evidence Theory", Acta Electronica Sinica, Vol. 39, No. 8, 2004, pp 611-628

[106] W. Zheng, D.-H. Lee, and Q. Shi,'Short-term freeway traffic flow prediction: Bayesian combined neural network approach". Journal of Transportation Engineering, Vol. 132, No. 2, pp.114-121, 2006.

[107] S.R. Chandra and H. Al-Deek, "Predictions of freeway traffic speeds and volumes using vector autoregressive models". Journal of Intelligent Transportation Systems, vol. 13, no. 2, pp.53-72, 2009.

[108] S. I. J. Chien, and C. M. Kuchipudi, "Dynamic travel time prediction with real-time and historical data", in: Proceedings of the Transportation Research Board 81st Annual Meeting, Washington, DC, 2002.

[109] A. Stathopoulos, and M. G. Karlaftis, "A multivariate state-space approach for urban traffic flow modelling and prediction", Transportation Research Part C, vol. 11, no. 2, pp. 121-135, 2003.

[110] Eriksson, Owen, and K. Axelsson. "ITS Systems ArchitecturesFrom Vision to Reality." PROCEEDINGS OF THE 7TH WORLD CONGRESS ON INTELLIGENT SYSTEMS. 2000.

[111] Blinov, Zuzana, Petr Bure, and Peter Jesty. "Intelligent transport system architecture different approaches and future trends." Data and Mobility. Springer Berlin Heidelberg, 2010. 115-125.

[112] "SOL - Save Our Lives. A Comprehensive Road Safety Strategy for Central Europe", project website, http://www.sol-project.eu/, 2010-2013

[113] "CO-OPerative SystEms for Intelligent Road Safety", project website, http://www.coopers-ip.eu/, 2006-2010

[114] Bonnefoi, Fabien, et al. "SAFESPOT Applications for Infrasructurebased Co-operative Road Safety." 14th World Congress and Exhibition on Intelligent Transport Systems and Services. 2007.

[115] "Connect \& Drive", project website, http://www.tue.nl/en/university/departments/industrialdesign/research/research-programs/user-centeredengineering/research/projects/explorations-in-interactions/connectdrive/, 2009-2011

[116] "Worldwide Cellular M2M Modules Forecast Market Brief," Beecham Research, Aug. 2010

[117] S. Lucero, "Maximizing Mobile Operator Opportunities in M2M: The Benefits of an M2M-Optimized Network," ABI Research, 1Q 2010.

[118] OECD- Machine-to-Machine Communications: Connecting Billions of Devices, OECD Digital Economy Papers, No. 192, 2012.

[119] Vreeswijk, J. D., M. K. M. Mahmod, and B. Van Arem. "Energy efficient traffic management and control-the eCoMove approach and expected benefits.'Intelligent Transportation Systems (ITSC), 2010 13th International IEEE Conference on. IEEE, 2010.

[120] "Combining Infrastructure for Efficient Electric Mobility (eCo-FEV)", project website, http://www.eco-fev.eu/, 2012-2015

[121] Toulminet, Gwenalle, Jacques Boussuge, and Claude Laurgeau. "Comparative synthesis of the 3 main European projects dealing with Cooperative Systems (CVIS, SAFESPOT and COOPERS) and description of COOPERS Demonstration Site 4." Intelligent Transportation Systems, 2008. ITSC 2008. 11th International IEEE Conference on. IEEE, 2008. "Cooperative Vehicle Infrastructure Systems (CVIS)", project website, http://www.cvisproject.org, 2006-2010

[122] "Geo-addressing and geo-routing for vehicular communications (GeoNET)", project website, http://cordis.europa.eu/projects/rcn/85551_en.html, 2008-2012

[123] Casimiro, A., Lollini, P., Dixit, M., Bondavalli, A., \& Verssimo, P. (2008, March). A framework for dependable QoS adaptation in probabilistic environments. In Proceedings of the 2008 ACM symposium on Applied computing (pp. 2192-2196). ACM.

[124] "Secure Vehicular Communications (SeVeCom)", project website, http://www.sevecom.org/ 2006-2010

[125] Amditis, Angelos, et al. "Design and development of an adaptive integrated driver-vehicle interface: overview of the AIDE project." Proceedings of the IFAQ conference, Prague. 2005.

[126] Roche-Cerasi, Isabelle, et al. "Road Safety Attributes Exchange Infrastructure in Europe: State of the Art and Perspectives." 16th ITS World Congress and Exhibition on Intelligent Transport Systems and Services. 2009.
[127] IEEE1609 WAVE Workgroup, http://vii.path.berkeley.edu/1609_wave/

[128] Vezin, P., et al. "5.15. European Biomechanical Experiment Database: A Tool to Promote the Sharing and the Dissemination of the Biomechanics Experiments with Human Subject for Passive Safety." D16-WG 5.2 Workshop on Biomechanical Experiments. 2004.

[129] KELLENDONK, G., and J. WISMANS. "Advanced Protection Systems (APROSYS)." TRA-TRANSPORT RESEARCH ARENA EUROPE 2006: GOETEBORG, SWEDEN, JUNE 12TH-15TH 2006: GREENER, SAFER AND SMARTER ROAD TRANSPORT FOR EUROPE. PROCEEDINGS (2006).

[130] TOMTOM Website, http://www.tomtom.com/

[131] GARMIN Website, http://www.garmin.com/

[132] http://www.technologyreview.com/news/515966/the-internet-of-cars-isapproaching-a crossroads/

[133] http://parkya.com/

[134] http://www.parkinglook.com.au/

[135] http://www.worldsensing.com/news-press.html

[136] D. Zhang, Y. Li, F. Zhang, M. Lu, Y. Liu, and T. He, "coRide:Carpool Service with a Win-Win Fare Model for Large-Scale Taxicab Networks", ACM SenSys 2013, Rome, Italy, pp. 1-14.

[137] D. Zhang, T. He, Y. Liu and J. A. Stankovic,"callCab:A Unified Recommendation System for Carpooling and Regular Taxicab Services", IEEE BIGDATA 2013, Santa Clara, CA, USA, pp. 439-447.

[138] A. Kumar, S. K. Gupta, A. K. Rai, and S. Sinha, "Social Networking Sites and Their Security Issues," International Journal of Scientific and Research Publications, vol. 3, no. 4, p. 3, 2013.

[139] K. Zhang, X. Liang, X. Shen, and R. Lu, "Exploiting multimedia services in mobile social networks from security and privacy perspectives," Communications Magazine, IEEE, vol. 52, no. 3, pp. 58-65, 2014.

[140] H. T. Cheng, H. Shan, and W. Zhuang, "Infotainment and road safety service support in vehicular networking: From a communication perspective", Mechanical Systems and Signal Processing, vol. 25, no. 6, pp. 2020-2038, Aug. 2011.

[141] C. Xu, F. Zhao, J. Guan, H. Zhang, and G.-M. Muntean, "QoE-Driven User-Centric VoD Services in Urban Multihomed P2P-Based Vehicular Networks," IEEE Transactions on Vehicular Technology, vol. 62, no. 5, pp. 2273-2289, Jun. 2013.

[142] R. V. D Heijden, S. Dietzel, F. Kargl, "Misbehavior Detection in Vehicular Ad-hoc Networks". Proceedings of the 1st GI/ITG KuVS Fachgesprch Inter-Vehicle Communication (FG-IVC 2013),Innsbruck, Tyrol, Austria, Feb. 21-22, 2013.

[143] C.-C. Lee and Y.-M. Lai, "Toward a secure batch verification with group testing for VANET," Wireless Networks, vol. 19, no. 6, pp. 14411449, Aug. 2013.

[144] Z. Zhang, P.-H Ho, and F. Nait-Abdesselam, "RADAR: A ReputationDriven Anomaly Detection System for Wireless Mesh Networks", ACM Wireless Networks (WINET), vol. 16, no. 8, pp. 2221-2236, 2010.

[145] S. Wang, S. Djahel J. McManis, C. McKenna and L. Murphy, "Comprehensive Performance Analysis and Comparison of Vehicles Routing Algorithms in Smart Cities”, IEEE GIIS 2013, Trento, Italy, 28-31 October 2013

[146] D. Smith, S. Djahel, and J. Murphy, "A SUMO Based Evaluation of Road Incidents' Impact on Traffic Congestion Level in Smart Cities", goSMART 2014, The 3rd IEEE International Workshop on GlObal Trends in SMART Cities, co-located with IEEE LCN 2014, Edmonton, Canada, Sep. 8-11, 2014.

[147] J. Li, Z. Zhang, and W. Zhang, "MobiTrust: Trust Management System in Mobile Social Computing", Proc. of the 3rd IEEE International Symposium on Trust, Security, and Privacy for Emerging Applications (TSP-10), Bradford, UK, Jun. 29-Jul. 01, 2010.

[148] C. Dwyer, S. R. Hiltz, and K. Passerini, "Trust and privacy concern within social networking sites: A comparison of Facebook and MySpace", Proc. of the Thirteenth Americas Conference on Information Systems, Keystone, Colorado, Aug. 09-12, 2007.

[149] J. Golbeck, "Trust and nuanced profile similarity in online social networks", ACM Transactions on the Web (TWEB), vol. 3, no. 4, pp. 1-33, Sep. 2009. 


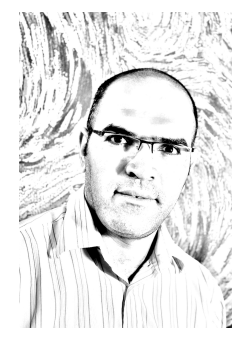

Dr. Soufiene Djahel is an engineering research manager at University College Dublin and member of wireless networking group of PEL since February 2012. Before joining in PEL, he was a postdoc fellow at ENSIIE where he was involved in a research project aiming at designing communication protocols for Hybrid Sensor and Vehicular Networks (HSVNs). He got his Ph.D degree in computer science in December 2010 from LILLE 1 UniversityScience and Technology of France. During his Ph.D, he was working on security issues at MAC and Routing layers in wireless multi-hop networks. Prior to that, he spent 6 months at INRIA NORD Europe research center as an engineer researcher. $\mathrm{He}$ received a Magister degree with majors in networking and distributed systems and a state engineering degree in computer science from Abderrahmanemira University (Bejaia, Algeria) and Badji-Mokhtar University (Annaba, Algeria) in February 2007 and June 2004, respectively. The research interests of Soufiene Djahel include Intelligent Transportation Systems, Security and QoS issues in Wireless Networks (VANETs, MANETs, WSNs and WBANs) and Internet of Things. He is member of IEEE and reviewer of its major conferences and journals in wireless networks and security. He was the general co-chair of VTM 2014 and the TPC co-chair of VTM 2012, and has served on the TPC of several conferences including IEEE ICC, IEEE WCNC, IEEE Globecom and IEEE IWCMC.

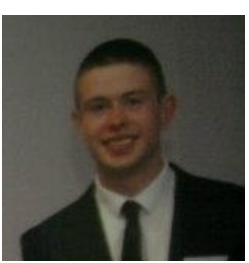

Ronan Doolan received his bachelors degree in Mechatronic Engineering from Dublin City University in Ireland in 2011. He is currently a PhD. Candidate in the performance engineering laboratory in DCU. He is funded by LERO the Irish software foundation (Grant no. 10/CE/11855). His research interests include Vehicular Ad Hoc Networks, Electric Vehicles, Vehicular Routing, Smart Cities.

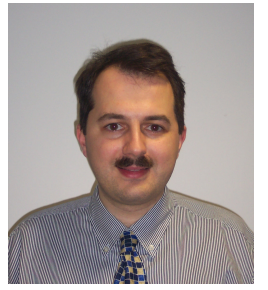

Dr. Gabriel-Miro Muntean is a Senior Lecturer with the School of Electronic Engineering, Dublin City University (DCU), Ireland, where he obtained his Ph.D. degree in 2003 for research on quality oriented adaptive multimedia streaming over wired networks. He was awarded the B.Eng. and M.Sc. degrees in Software Engineering from the Computer Science Department, "Politehnica" University of Timisoara, Romania in 1996 and 1997 respectively. Dr. Muntean is co-Director of the DCU Performance Engineering Laboratory and Consultant Professor with the Beijing University of Posts and Telecommunications, China. His research interests include quality-oriented and performance-related issues of adaptive multimedia delivery, performance of wired and wireless communications and energy-aware networking. Dr. Muntean has published over 180 papers in prestigious international journals and conferences, has authored three books and sixteen book chapters and has edited six other books and conference proceedings. Dr. Muntean is Associate Editor of the IEEE Transactions on Broadcasting, Associate Editor for the IEEE Communication Surveys and Tutorials and reviewer for other important international journals, conferences and funding agencies. He is a member of IEEE, ACM and IEEE Broadcast Technology Society.

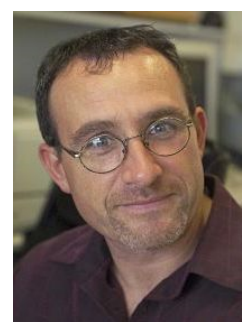

John Murphy is an Associate Professor in Computer Science and Informatics at University College Dublin. He received a first class honours degree in electronic engineering (B.E.) in 1988 from the National University of Ireland (UCD), an M.Sc. in electrical engineering from the California Institute of Technology in 1990 and a Ph.D. in electronic engineering from Dublin City University in March 1996. He is an IBM Faculty Fellow, a Fellow of the Institution of Engineering and Technology, a Senior Member of the IEEE, a Fellow and Chartered Engineer with Engineers Ireland, and a Fellow of the Irish Computer Society. For many years he held an academic part-time position at the Jet Propulsion Laboratory in Pasadena, and acted as a consultant to the US Department of Justice.

Prof. Murphy is an editor for both IEEE Communications Surveys and Tutorials (since 2012) and Telecommunications Systems Journal (since 2008), and a guest editor for an upcoming issue on 'Automation in Software Performance Engineering' in the Automated Software Engineering Journal (2014). He has served on the Editorial Board of IEEE Communications Letters (2008-2012) and IET Communications (2006-2010), where he was a guest editor (with Prof. Perros) for 'Optical Burst and Packet Switching' in 2009. $\mathrm{He}$ has published over 100 peer-reviewed journal articles or international conference full papers in performance engineering of networks and distributed systems. He has supervised $17 \mathrm{Ph} . \mathrm{D}$. students to completion and been awarded over 20 competitive research grants (in excess of 7.5 million euro) 